No. 20-16

\title{
Consumption Heterogeneity by Occupation: Understanding the Impact of Occupation on Personal Consumption during the COVID-19 Pandemic
}

\section{Christopher D. Cotton, Vaishali Garga, and Justin Rohan}

\begin{abstract}
:
This paper exploits the variation in the unemployment rate of different occupations in the first part of the COVID-19 pandemic to analyze the response of consumption spending to unemployment risk. We find that earlier in the pandemic, higher unemployment risk did not reduce relative spending. However, as the pandemic proceeded, higher unemployment risk reduced relative spending. This pattern held across both essential and nonessential spending categories. We find that "high-risk" occupations had three common characteristics: lower ability to be performed from home, higher physical proximity on the job, and a nonessential nature.
\end{abstract}

JEL Classifications: E21, E32, E62

Keywords: consumption, occupation, unemployment risk, COVID-19, CARES Act

Christopher D. Cotton and Vaishali Garga are economists in the research department of the Federal Reserve Bank of Boston; their emails are Christopher.Cotton@bos.frb.org and Vaishali.Garga@bos.frb.org, respectively. Justin Rohan is a research assistant in the Boston Fed's research department; his email is Justin.Rohan@bos.frb.org.

The authors thank Daniel Cooper, María Luengo-Prado, and Giovanni Olivei for comments.

This paper presents preliminary analysis and results intended to stimulate discussion and critical comment.

The views expressed herein are those of the authors and do not indicate concurrence by the Federal Reserve Bank of Boston, the principals of the Board of Governors, or the Federal Reserve System.

This paper, which may be revised, is available on the website of the Federal Reserve Bank of Boston at https://www.bostonfed.org/publications/research-department-working-paper.aspx. 


\section{Introduction}

The COVID-19 pandemic presents a significant health threat to the United States, with reported cases numbering more than 10 million and the disease's death toll surpassing 240,000 at the time of writing. In response, the government has enacted a variety of policy interventions to limit the spread of the virus, such as social-distancing and lockdown measures. These mitigation strategies seek to prevent community transmission of the virus by limiting the size of gatherings, ensuring a minimum amount of physical distance between individuals in public, and closing down "nonessential" elements of the economy. These strategies are not without cost - they have impacted the U.S. economy in unprecedented ways. At the start of the crisis in April, the unemployment rate spiked to 14.7 percent - its highest level since the Great Depression. Four months later, the August unemployment rate remained well above average levels at 8.4 percent.

Importantly, these interventions to limit the spread of COVID-19 did not impact all occupations equally during the first part of the pandemic. On the one hand, there were jobs that could be performed virtually from home without any productivity losses (for example, computer-related and mathematical occupations) and jobs that, though unable to be performed from home, were "essential" and thereby exempt from the strict lockdown requirements (for example, health-care occupations). On the other hand, there were jobs that could not be performed from home but were not essential, so workers in these occupations bore a disproportionately high cost of the mitigation strategies in terms of unemployment risk (for example, food-service occupations). To help mitigate this economic fallout, the government passed the Coronavirus Aid, Relief, and Economic Security (CARES) Act. This $\$ 2.2$ trillion relief package included expanded unemployment insurance (UI) benefits and Economic Impact Payments (or stimulus payments) to households with income of less than $\$ 99,000$, and these benefits varied over time.

In this paper, we use data on high-frequency credit/debit card spending to investigate how consumption spending varied by occupation during the pandemic. We are particularly interested in analyzing whether workers in occupations that faced higher risk of unemployment as a result of the pandemic-induced structural changes demonstrated relatively lower consumption spending driven by a precautionary motive.

We divide the pandemic sample period into five five-week sub-periods. They include a "prestimulus" period from March 9 through April 12, 2020, a "post-stimulus" period from July 27 through August 30, 2020, and three five-week periods in between. The stimulus checks under the CARES Act were posted during the week of April 13 through 20, so the pre-stimulus period was chosen to obtain a clean estimate of the effect of occupation on spending that is not muddled by stimulus payments, which we are unable to track (since we don't observe individual income or savings in our data). Expanded UI benefits under the CARES Act were available for all weeks through Friday, July 31. However, because states' unemployment weeks typically end on Saturday/Sunday, July 26 marked the end of when these expanded UI benefits were available. So the post-stimulus period was chosen to facilitate a comparison of the estimates from this period with the estimates from the pre-stimulus period to shed light on the effectiveness of the CARES Act.

We establish five novel facts regarding the effect of occupational unemployment risk on consumption spending. First, occupational unemployment risk appears to have a positive impact on 
relative consumption spending in the pre-stimulus period. This relationship implies that workers in occupations with higher unemployment risk were spending relatively more or at least not spending less than their less risky counterparts. This result is counterintuitive in light of the prediction of a standard economic model of consumption. Inter-temporal consumption smoothing based on an Euler equation predicts that expectation of future unemployment, which is equivalent to a future income loss, should lead to a reduction in consumption spending today. One potential explanation for why spending among the workers in riskier occupations was relatively higher is that the workers were anticipating stimulus payments and unemployment benefits, which in a large number of cases were higher than their regular salaries. While these benefits were not paid out until mid-April, they were authorized under the CARES Act on March 27 and therefore anticipated as of that date. Another explanation could be that workers' perceptions regarding the unemployment risk of their occupation were not in line with the realized unemployment rates.

Second, occupational unemployment risk appears to drive lower relative consumption in the post-stimulus period. There are several possible reasons why we observe this reversal in spending behavior later in the pandemic: (1) it is possible that actual income was changing during this period as government benefits were largely reduced, thereby necessitating a cut in spending; (2) it is possible that workers' perceptions of the unemployment risk evolved with the progression of the pandemic. Persistence of unemployment several months into the pandemic may have led workers to revise their expectations of future job loss and driven them to reduce relative spending.

Third, we find that occupational unemployment risk has an increasingly negative effect on relative consumption spending month by month as the pandemic proceeds. We verify this by looking at the relationship between occupational unemployment risk and consumption on a monthby-month basis during the pandemic. We find that as each month passes, the relationship becomes progressively more negative. This fits our broader story. As the hopes of further stimulus receded, and the likelihood of longer-term unemployment risk rose, workers facing greater occupational unemployment risk were likely to re-evaluate their consumption plans.

Fourth, we consider the results disaggregated by spending across essential (grocery, utility) versus nonessential (recreation, food-out) categories. We find that in the pre-stimulus period, occupational unemployment risk has a negligible effect on relative spending across all categoriesthis effect is positive for grocery and negative for the remaining three categories. In the poststimulus period, however, unemployment risk has a large negative impact on relative spending across all categories.

Fifth, we create a new metric, based on job characteristics, to predict which occupations were likely to face higher rates of unemployment in the COVID-19 pandemic. We find that the occupations in which workers were more likely to become unemployed during this time have three common characteristics. First, they cannot be performed from home. Those occupations in which business simply can be conducted virtually did not experience significant unemployment. Second, they tend to be occupations in which workers are in close proximity to one another, making abiding by social-distancing policies more difficult. Third, they tend to be less "essential" occupations, meaning they could be more easily shut down without having a large immediate impact on society. As examples, both health-care workers and food-service workers may not be able to work from home and are required to work in close proximity to other people. However, health-care workers 
were more likely to keep working during the pandemic, as their role was judged to be more vital for society. Our measure appears to perform better than existing measures at predicting unemployment rates.

Related Literature Our work contributes to the existing literature in three main ways. First, it contributes to the recent literature analyzing the behavior of household consumption following the onset of the COVID-19 pandemic and its interaction with households' macroeconomic expectations and stimulus payments from the government. Baker et al. (2020a) find that there was an initial spike in consumption for certain product categories (groceries, retail, and credit card spending) that was followed by a significant decline in overall spending. Coibion, Gorodnichenko, and Weber (2020) find a similar decline in overall spending, which they attribute mostly to various lockdown measures. Baker et al. (2020b) find that after the CARES Act payments began to be distributed in April, there was a significant increase in spending, especially among individuals who had lower incomes or had experienced greater decreases in income as result of the pandemic. Casado et al. (2020) estimate that eliminating the expanded unemployment payments from the CARES Act would have resulted in a 44 percent decrease in local spending. While these papers analyze the impact of the pandemic on overall spending, our paper studies the heterogeneity in the spending response for workers in different occupations with a focus on the role of unemployment risk in driving spending responses.

Second, we add a new data point to the existing literature on the degree to which the expectation of future unemployment affects current spending. Existing papers measure this expectation in two main ways: using survey-based subjective probabilities of unemployment reported directly by the respondents (Stephens (2004); Karahan, Moore, and Pilossoph (2019)) or by proxying for the ex ante expectation of unemployment using ex post realized unemployment (Hendren (2017); Basten, Fagereng, and Telle (2016)). The novelty of our paper is that we exploit the unique circumstances provided by the COVID-19 pandemic, in which occupation can be used as a proxy for unemployment risk. There is considerable exogenous variation in the degree to which workers in different occupations were differentially exposed to the risk of unemployment, owing to the nature of the virus and the non-pharmaceutical policy interventions enacted in response to it. In a similar spirit, Skinner (1988) uses occupation as a proxy for earnings risk but finds no evidence that workers in riskier jobs save more.

Finally, our new metric of unemployment risk based on job characteristics is an improvement over the existing metrics used in the pandemic literature. Following Dingel and Neiman (2020), Leibovici, Santacreu, and Famiglietti (2020), Montenovo et al. (2020), and Mongey, Pilossoph, and Weinberg (2020), we assign scores to different occupations based on the ability of employees to work from home as well as how frequently an employee is required to work in close physical proximity with others. However, using these two scores alone misses an important aspect of unemployment risk during the pandemic - namely, the degree to which jobs are considered vital for society. We introduce a third, new score to measure this aspect of different occupations. We combine these three scores to create a binary categorization of occupations as being high risk or not and show that our metric does a better job of predicting unemployment rates compared with the existing metrics used in the literature. 
The rest of the paper proceeds as follows. Section 2 discusses the data used in the analysis. Section 3 contains the main empirical specification and results regarding the impact of unemployment risk on consumption spending during various phases of the pandemic. Section 4 discusses our alternative measure of unemployment risk based on job characteristics. Section 5 concludes.

\section{Data}

In analyzing the effect of unemployment risk on consumption spending during the pandemic, we use data from two main sources: Affinity Data Solutions (Affinity) and the American Community Survey (ACS). In creating the alternative metric to measure unemployment risk based on job characteristics, we use data from the Occupational Information Network (O*NET).

Affinity Affinity has relationships with many banks and financial institutions for marketing purposes, and as part of this arrangement Affinity has access to detailed information on credit/debit card transactions for members of these institutions. Affinity's data set measures activity on a sample of 40 million active cards that captures 6 to 9 percent of all card transactions in the United States. ${ }^{1}$ These data are aggregated and available at various levels of geographic and spending-category disaggregation. In particular, we have weekly data on spending at the Zip code level by Merchant Classification Category (MCC) - the worldwide classification system used by Visa and other credit card providers that assigns a transaction category to each merchant. In terms of geography, the sample is fairly representative, with the mid-Atlantic states being slightly underrepresented. ${ }^{2}$ In terms of age, conditional on age being known and adjusting for the number of unique cards for a given age group in a spending category at a point in time, roughly 17 percent of the sample is 18 to 34 years old, 40 percent is 35 to 54 years old, and 42 percent is 52 or older. Finally, in terms of income, 5 percent of the sample earns $\$ 0$ to $\$ 35 \mathrm{~K}, 50$ percent earns $\$ 35 \mathrm{~K}$ to $\$ 85 \mathrm{~K}$, and 45 percent earns more than $\$ 85 \mathrm{~K}$ annually. Thus, the sample comprises the relatively affluent banked population. ${ }^{3}$

Since we observe only high-frequency card spending, we miss cash transactions and other transactions that don't involve payment by cards. These include payments for health procedures that go directly through the insurance company, for home mortgages, rent, auto purchases, and payments to contractors who accept only cash.

ACS While the Affinity data provide weekly Zip-code-level card spending, there is no employment information in this data set. For this reason, we combine the data with employment information from the 2014-2018 ACS 5-year sample. The lowest level of geography that is easily compatible with the Affinity data and for which employment information is available in the ACS is a Zip Code Tabulation Area (ZCTA). ${ }^{4}$ Thus, we aggregate the Zip-code-level spending data from

\footnotetext{
${ }^{1}$ According to our back-of-the-envelope calculations based on Kumar and O'Brien (2019), this represents 3.06 to 4.59 percent of the total transactions in the United States.

${ }^{2}$ Comparing the share of spending by region in the Affinity sample versus the 2017 Economic Census, we find that the mid-Atlantic states account for 14.58 percent of spending in the census compared with 8.29 percent in the Affinity sample.

${ }^{3}$ We thank Daniel Cooper for providing these details on the representativeness of the Affinity sample.

${ }^{4}$ Zip code is a trademark of the U.S. Postal Service, whereas ZCTA is the Census Bureau's measure of a Zip code. The main difference between them is that while Zip codes can correspond to stand-alone buildings and often
} 
Affinity to the ZCTA level using a crosswalk provided by the U.S. Census Bureau.

At the ZCTA level, the most precise measure of occupation available are SOC major groups, which are defined by a two-digit code and sort an occupation into one of 22 categories. These categories include occupations such as "personal care and service," "legal," and "protective services."

We also obtain ZCTA-level demographic and economic data on age, sex, race, median income, and educational attainment from the same ACS sample.

Finally, we supplement this information with data from the 2010 Census measuring the percentage of the population in a ZCTA that is living in rural/urban regions, as well as results from the 2016 presidential election provided by the MIT Election Lab.

O*NET The O*NET, under the sponsorship of the U.S. Department of Labor, gathers data on nearly 1,000 occupations ${ }^{5}$ by conducting a series of surveys with members of these occupations. For our purpose, we use data from the O*NET 24.3 Work Context and Work Activities surveys. Further details on this data are provided in Section 4.

\section{Impact of Occupation on Consumption}

In this section, we examine how consumption spending varied by occupation during the first part of the pandemic. We are particularly interested in analyzing whether workers in occupations that faced higher unemployment risk as a result of the pandemic-induced structural changes demonstrated relatively lower consumption spending driven by a precautionary motive.

\subsection{Empirical Specification}

Our empirical approach, summarized in equation (1), is to examine how the consumption spending of a ZCTA with a higher share of a given occupation compares with a similar ZCTA with a lower share of that occupation before and during the pandemic. The dependent variable is the log of spending by consumers residing in a ZCTA $z$ in week $t$. We use the log of spending to look at the percentage change in spending across ZCTAs, which avoids the problem of differences in size across ZCTAs. We also include ZCTA fixed effects $\left(\alpha_{z}\right)$ and weekly time fixed effects $\left(\beta_{t}\right)$ in all our regressions.

$$
\begin{aligned}
\log \_ \text {spending }_{z, t} & =\alpha_{z}+\beta_{t}+\sum_{j} \gamma_{j}\left(\text { postcovid }_{t} \times \text { occupation }_{z}^{j}\right)+\delta_{d}\left(\text { postcovid }_{t} \times \text { demographic }_{z}\right) \\
& +\delta_{e}\left(\text { postcovid }_{t} \times \text { economic }_{z}\right)+\eta_{s}\left(\text { postcovid }_{t} \times \text { state }_{z}\right)+u_{z, t}
\end{aligned}
$$

The primary variable of interest is $\left(\right.$ postcovid $_{t} \times$ occupation $\left._{z}^{j}\right)$. postcovid $_{t}$ is a dummy variable for whether or not the time period takes place after the economic effects of COVID-19 began to be felt in the United States. It is set to be 1 for weeks beginning on or after March 9, 2020, which was chosen because this was the date when COVID-19 was declared a national emergency in the

change over time, ZCTAs are slightly less disaggregated but tend to be stable over time. There are 42,000 Zip codes versus 32,000 ZCTAs in the United States.

${ }^{5} \mathrm{O} * \mathrm{NET}$ classifies occupations into a taxonomy based on 2010 SOC detailed occupation codes that it further divides into what it refers to as $\mathrm{SOC}-\mathrm{O}^{*} \mathrm{NET}$ occupations. 
United States and significant restrictions began to be put in place to combat the virus. occupation oc $_{z}$ represents the proportion of people in a ZCTA $z$ working in occupation $j$.

There are 22 possible occupations, which represent each of the two-digit SOCs. We include the post-COVID occupation interaction for all but one of the occupation groups to avoid the problem of multicollinearity. We omit the occupation group "protective services," which includes occupations such as correctional officers, firefighters, and police. ${ }^{6}$ ( postcovid $_{t} \times$ occupation $\left._{z}^{j}\right)$ represents the percentage increase in the consumption of a ZCTA with a 1 percentage point (p.p.) higher share of occupation $j$ and a 1 p.p. lower share of protective services occupations in post-COVID weeks relative to pre-COVID weeks. Since all of these coefficients are relative to protective services, the absolute magnitude of the coefficients is unimportant; instead we are interested only in the relative size of the coefficients across different occupations.

We include several controls. First, we control for the interaction between various demographic controls and the post-COVID dummy ( postcovid $_{t} \times$ demographic $\left._{z}\right)$. These demographic controls are the share of the ZCTA population over age 55; the share of the ZCTA that identifies as male; the share of the ZCTA that identifies as Black, Asian-American, multiple races, and other nonwhite races; the share of the ZCTA that lives in a rural area; the share of the ZCTA's county that voted for the Republican party in the 2016 presidential election; and the share of the ZCTA's county that voted for a third party. These terms capture the fact that the consumption spending of different demographic groups may differ relatively after the onset of the pandemic compared with beforehand. We also sometimes control for interactions between economic variables and the post-COVID dummy $\left(\right.$ postcovid $_{t} \times$ economic $\left._{z}\right)$. The economic controls we use are the median income of the ZCTA and the share of people in a ZCTA who are enrolled in college. Finally, we include an interaction term between the state a ZCTA is located in and the post-COVID dummy $\left(\right.$ postcovid $_{t} \times$ state $\left._{z}\right)$ to control for any common changes in consumption spending across a state. ${ }^{7}$

\subsection{Pre-stimulus}

To start, we focus on the five-week period after the start of the pandemic and before the impact of the CARES Act was felt-March 9 through April 12, 2020. We compare this five-week, postCOVID, pre-stimulus period to the pre-COVID period (January 1 through March 8, 2020).

\footnotetext{
${ }^{6}$ The reason we use protective services as our omitted category is because it has a fairly average education and income level compared with other occupations, as can be seen in Table 1.

${ }^{7}$ Appendix A.1 provides a discussion of how introducing these controls affects the results.
} 
Table 1. Economic Controls and Unemployment Figures by Occupation

\begin{tabular}{|c|c|c|c|c|c|}
\hline & $\begin{array}{c}(1) \\
\text { Log Spend }\end{array}$ & Median Annual Wage & Percent with Some College Degree & $\begin{array}{c}\text { April YoY } \\
\text { Change in Unemployment }\end{array}$ & $\begin{array}{c}\text { August YoY } \\
\text { Change in Unemployment }\end{array}$ \\
\hline Installation, Maintenance, and Repair Occupations & $\begin{array}{c}0.306 \\
(0.0573)\end{array}$ & 46,630 & 0.21 & 10.10 & 4.10 \\
\hline Office and Administrative Support Occupations & $\begin{array}{c}0.294 \\
(0.0603)\end{array}$ & 37,580 & 0.35 & 9.30 & 3.20 \\
\hline Production Occupations & $\begin{array}{c}0.293 \\
(0.0604)\end{array}$ & 36,000 & 0.16 & 14.80 & 5.80 \\
\hline Food Preparation and Serving Related Occupations & $\begin{array}{c}0.255 \\
(0.0636)\end{array}$ & 24,220 & 0.18 & 36.90 & 16.70 \\
\hline Healthcare Support Occupations & $\begin{array}{c}0.228 \\
(0.0665)\end{array}$ & 28,470 & 0.29 & 9.80 & 5.20 \\
\hline Transportation and Material Moving Occupations & $\begin{array}{c}0.215 \\
(0.0570)\end{array}$ & 32,440 & 0.16 & 13.90 & 6.40 \\
\hline Healthcare Practitioners and Technical Occupations & $\begin{array}{c}0.207 \\
(0.0790)\end{array}$ & 68,190 & 0.79 & 5.60 & 2.00 \\
\hline Legal Occupations & $\begin{array}{c}0.205 \\
(0.0832)\end{array}$ & 81,820 & 0.86 & 2.60 & 3.00 \\
\hline Construction and Extraction Occupations & $\begin{array}{c}0.179 \\
(0.0641)\end{array}$ & 47,430 & 0.13 & 13.70 & 4.00 \\
\hline Farming, Fishing, and Forestry Occupations & $\begin{array}{c}0.127 \\
(0.0573)\end{array}$ & 27,180 & 0.11 & 1.90 & 3.00 \\
\hline Arts, Design, Entertainment, Sports, and Media Occupations & $\begin{array}{c}0.101 \\
(0.0605)\end{array}$ & 51,150 & 0.72 & 14.70 & 8.70 \\
\hline Sales and Related Occupations & $\begin{array}{c}0.0618 \\
(0.0548)\end{array}$ & 29,630 & 0.39 & 13.20 & 4.80 \\
\hline Personal Care and Service Occupations & $\begin{array}{l}0.00919 \\
(0.0559)\end{array}$ & 26,220 & 0.31 & 35.60 & 9.50 \\
\hline Computer and Mathematical Occupations & $\begin{array}{l}-0.0218 \\
(0.0866)\end{array}$ & 88,340 & 0.79 & 1.90 & 3.10 \\
\hline Architecture and Engineering Occupations & $\begin{array}{c}-0.0336 \\
(0.0956)\end{array}$ & 81,440 & 0.77 & 3.70 & 2.90 \\
\hline Life, Physical, and Social Science Occupations & $\begin{array}{l}-0.0826 \\
(0.0730)\end{array}$ & 68,160 & 0.86 & 4.80 & 1.20 \\
\hline Building and Grounds Cleaning and Maintenance Occupations & $\begin{array}{c}-0.114 \\
(0.0772)\end{array}$ & 28,330 & 0.13 & 17.30 & 6.40 \\
\hline Educational Instruction and Library Occupations & $\begin{array}{c}-0.117 \\
(0.0839)\end{array}$ & 50,790 & 0.81 & 12.60 & 5.30 \\
\hline Community and Social Service Occupations & $\begin{array}{l}-0.118 \\
(0.121)\end{array}$ & 46,090 & 0.78 & 2.60 & 1.40 \\
\hline Management Occupations & $\begin{array}{c}-0.150 \\
(0.0537)\end{array}$ & 105,660 & 0.61 & 5.20 & 2.70 \\
\hline Business and Financial Operations Occupations & $\begin{array}{c}-0.520 \\
(0.0790)\end{array}$ & 69,820 & 0.75 & 4.00 & 3.00 \\
\hline Protective Service Occupations & - & 41,580 & 0.42 & 6.90 & 2.50 \\
\hline
\end{tabular}

At the start of the pandemic, occupations with high unemployment risk had relatively higher consumption spending. Column 2 of Table 1 shows the impact of raising the share of an occupation by 1 p.p. on consumption spending using our basic empirical approach. Importantly, a positive coefficient for a post-COVID occupation interaction implies that ZCTAs with a higher share of this occupation spent relatively, not absolutely, more following the start of the pandemic. More specifically, the coefficient of 0.306 for installation occupations implies that a ZCTA with 1 p.p. more installation occupations workers spent 0.306 percent more than another ZCTA with 1 p.p. more protective services occupations workers after the start of the pandemic, all else being equal. Therefore, we see that installation, maintenance, and repair occupations spent the most, while business and financial operations occupations spent the least, in relative terms. Column 5 of Table 1 shows the change in unemployment from April 2019 to April 2020 by occupation. None of the top six occupations associated with the relatively highest increase in consumption spending has a change in unemployment of less than 6 p.p., while six of the bottom eight do. This is surprising, since it might have instead been expected that a greater share of higher unemployment occupations in a ZCTA would result in relatively lower consumption spending.

Graphically, we observe the same positive relationship between unemployment risk and relative 
consumption spending in the early part of the pandemic. To aid our analysis, we plot unemployment risk (the year-over-year change in April 2020 unemployment) against our relative spending regression coefficients in Figure 1(a.i). The positive relationship again implies that higher unemployment risk was associated with higher consumption spending early in the pandemic. 
Figure 1. Relationship between Unemployment Risk and Relative Spending during All Time Periods

\section{No Economic Controls With Economic Controls}
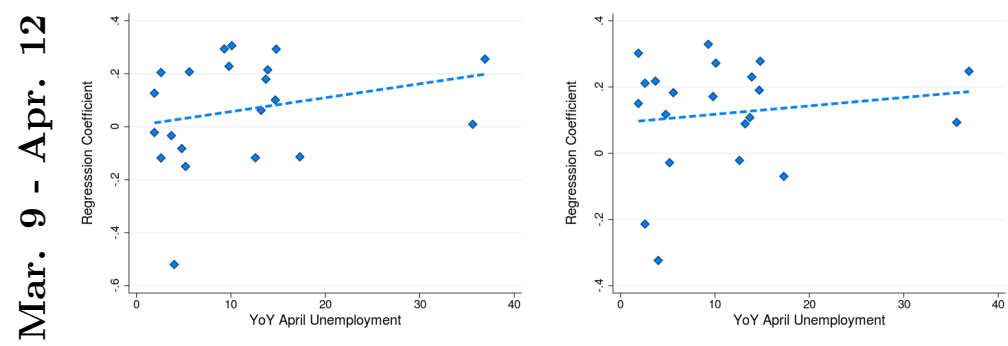

(a.i) Correlation: 0.249

(a.ii) Correlation: 0.146
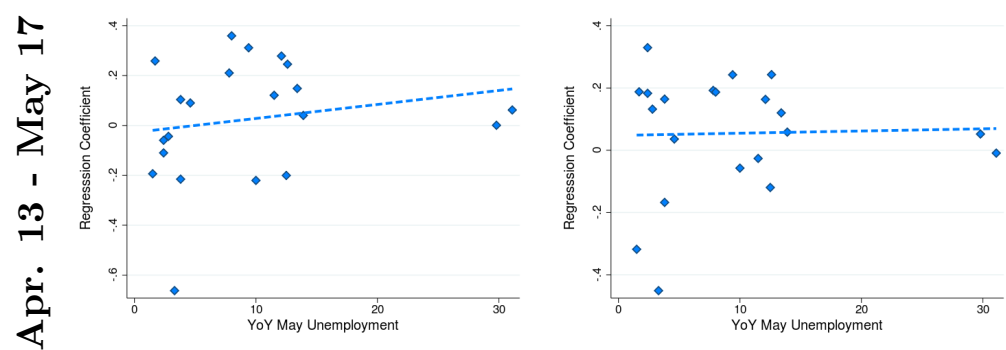

(b.i) Correlation: 0.192

(b.ii) Correlation: 0.030
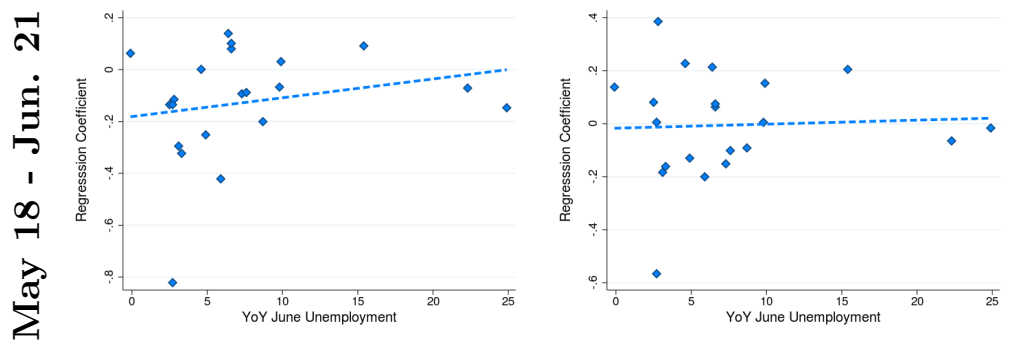

(c.i) Correlation: 0.210

(c.ii) Correlation: 0.047
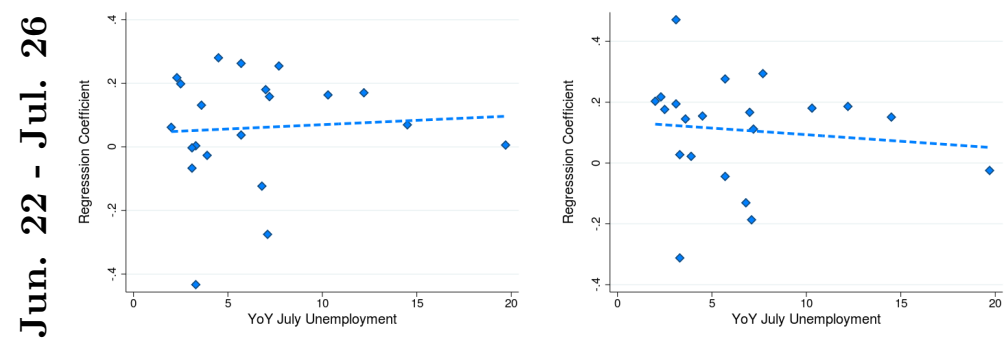

(d.i) Correlation: 0.068

(d.ii) Correlation: -0.110
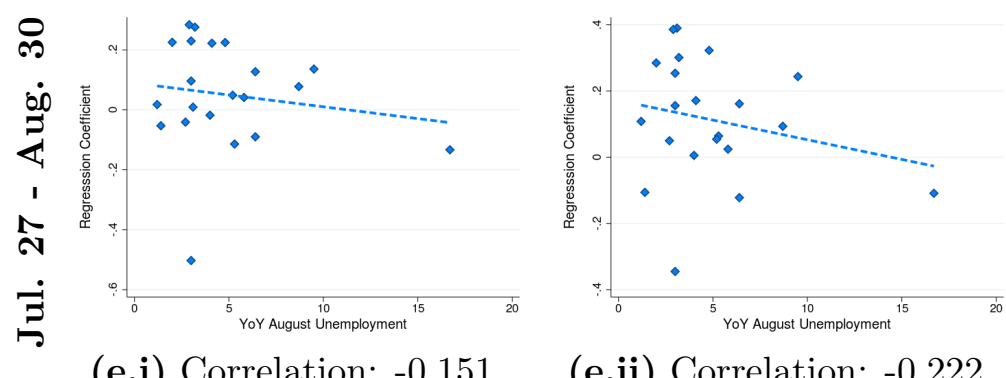

(e.ii) Correlation: -0.222

Sources: Unemployment figures from CPS, coefficients from authors' calculations using population data from the ACS and transaction data from Affinity.

It may be that the correlation of unemployment risk with other economic features of occu- 
pations, such as income and education levels, is driving the surprising result that occupations with high unemployment at the start of the pandemic had relatively high consumption spending. Columns 3 and 4 of Table 1 show the median annual wage and the share of workers with a college degree in each occupation. None of the top six occupations associated with the relatively highest increase in consumption spending has a median annual wage above $\$ 50 \mathrm{~K}$, while six of the bottom eight do. Similarly, none of the top six occupations associated with the relatively highest increase in consumption spending has a share of more than 50 percent of college-educated workers, while seven of the bottom eight do. Indeed, we find occupations with higher consumption spending earlier in the pandemic have lower wages and lower levels of education.

Even after controlling for economic factors, we find a positive association between occupational unemployment risk and relative consumption spending. To test whether economic factors are causing the positive relationship between unemployment risk and consumption spending, we introduce the economic controls discussed in Section 3.1 into the regression ${ }^{8}$. We present the results in Figure 1(a.ii) using the same approach as before. We again find a positive relationship between unemployment risk and the relative spending coefficients once economic controls are included. This relationship implies that workers in occupations with higher unemployment risk are still spending relatively more or, at least, are not spending less than their less risky counterparts. This result is surprising, as it means we find no evidence of precautionary cuts in spending in response to increased unemployment risk early in the pandemic.

We also find the same result with a longer-term measure of unemployment risk. We have so far measured the unemployment risk faced by workers in April 2020 as the year-over-year change in unemployment from April 2019 to April 2020. In column 6 of Table 1, we instead consider a longer-term unemployment risk measure of the year-over-year change in unemployment from August 2019 to August 2020. The operating assumption here is that workers' perceptions regarding the longer-term unemployment risk of their occupations were roughly consistent with the ex post realized unemployment rate of their occupation in August 2020. We again find the surprising result that workers with higher occupational risk as measured by this longer-term measure have higher consumption. ${ }^{9}$

Overall, we find no evidence that workers in occupations with higher unemployment risk spent relatively less early in the pandemic. This holds with both our short-term and long-term measures of unemployment risk and both with and without economic controls. We posit four possible explanations for why we observe this counterintuitive result. One: many low-wage workers may have anticipated receiving more in unemployment benefits than their usual salaries. Ganong, Noel, and Vavra (2020) estimate that 69 percent of workers were receiving unemployment benefits that were greater than their usual salaries including non-wage compensation. Two: workers may have initially underestimated the increase in unemployment risk resulting from the pandemic. Three: workers may have taken on credit card debt to smooth consumption. Four: we may be capturing early responses to stimulus payments. While we use April 12, 2020, as the start of CARES Act benefits, the act itself was signed into effect on March 27, meaning that from March 27 to April 12 individuals knew that they would be receiving money from the government, which in turn

\footnotetext{
${ }^{8}$ Results are given in regression (1) of the Appendix Table 4.

${ }^{9}$ Graphically, we depict this relationship in Figure 3 in Section A.4 of the Appendix.
} 
could have supported consumption spending. Additionally, it is unclear exactly when unemployed workers started receiving the additional $\$ 600$ benefit in unemployment insurance, though anecdotal evidence suggests that these payments were typically received after April 12.

\subsection{Post-Stimulus}

We study whether the continued pandemic and reduced government support affected how consumption spending varied with occupational unemployment risk. We focus on the five-week period after the expiration of CARES Act benefits-July 27 through August 30, 2020. As part of the CARES Act, direct payments to individuals were made once during April 2020, while expanded unemployment benefits effectively extended until July 26. From then on, unemployed individuals in participating states may still have been able to access some level of expanded unemployment insurance through the Lost Wages Assistance Program, but this amount was $\$ 200$ to $\$ 300$ a week less than under the CARES Act, and it was available under a stricter set of conditions. Therefore, this five-week period marked a substantial decrease in government support.

It appears that higher occupational unemployment risk lowered relative consumption spending as the pandemic continued. We apply the same approach as before to study how occupational unemployment risk affected consumption. ${ }^{10}$ We therefore use the same regression structure, except now the only post-pandemic weeks we include in the regression are the five weeks from July 27 through August 30. In Figure 1[e.i] and Figure 1[e.ii], we plot the August year-over-year change in unemployment for each occupation against its relative spending coefficient without and with economic controls, respectively. We find a negative relationship between occupational unemployment and relative consumption spending coefficients both without and with economic controls.

Multiple factors can explain the change in the pattern of consumption spending as the pandemic continued. First, it is possible that workers originally misjudged the change in unemployment risk. Workers may initially have thought that if they lost their job as a result of the pandemic they would quickly get it back. However, after many months, they may have decided that this was less likely and began to make precautionary cuts in spending. Second, instead of a change in risk, it might be that actual incomes were lower in this period, as unemployment benefits were reduced and stimulus was curtailed, meaning it may have been necessary for workers to cut back their spending.

\subsection{All Time Periods}

We break down the pandemic into five five-week periods and study how the relationship evolved across these five periods. We use the same method as before to analyze the results. The periods are March 9 through April 12, April 13 through May 17, May 18 through June 21, June 22 through July 26, and July 27 through August 30. We run the same regression both without and with economic controls. ${ }^{11}$ We exclude data points from after March 9 that are not included in the period we are examining; for example, when studying the April 13 through May 17 period, we include only data from before March 9 and the April 13-May 17 period in the regression. We

\footnotetext{
${ }^{10}$ Results are given in the Appendix A.2.

${ }^{11}$ The coefficients are given in the Appendix A.2.
} 
then compare these spending coefficients against the year-over-year change in unemployment in April, May, June, July, and August, respectively, in Figure 1. The graph letters represent the five-week periods from earliest to latest, while the graph numbers i and ii represent without and with economic controls, respectively. We already considered the first five-week period graphs and the last five-week period graphs in the pre-stimulus and post-stimulus analysis, respectively.

We find that occupational unemployment risk had an increasingly negative effect on consumption spending as the pandemic proceeded. In the pre-stimulus period, there was a positive relationship between spending and unemployment risk both without and with economic controls. During the stimulus periods, the positive coefficient lessened both without and with economic controls. By the end of the stimulus period, the relationship was mildly positive and mildly negative without and with economic controls, respectively. In the post-stimulus period, there was a negative relationship both without and with economic controls. Overall, we find that the positive relationship between occupational unemployment risk and consumption decreased in a noticeably smooth manner during the pandemic, both without and with economic controls.

The evolution of the relationship between consumption spending and occupational unemployment risk during the first part of the pandemic fits our broader story. At the start of the pandemic, workers in high-risk occupations did not cut their relative consumption spending, likely due to some combination of an anticipated high stimulus payment and imperfect perceptions of unemployment risk. Later in the pandemic, perhaps as perceptions of unemployment risk evolved, workers in these high-risk occupations gradually began to reduce their spending relative to other occupations. In the last period we study, workers in high-risk occupations cut their relative spending further as unemployment benefits were reduced and no future stimulus payments were promised or provided.

\subsection{Disaggregated by Spending Category}

To better understand the relationship between occupational unemployment risk and spending during the pre- and post-stimulus time periods, we examine how consumption varied by disaggregated spending categories.

We consider four separate categories of spending: grocery, utilities, food-out, and recreation. We select these specific categories as they cover "essential" (non-discretionary) spending on items such as groceries and utilities, as well as "nonessential" (discretionary) spending on food-out and recreation. The Affinity data enable us to perform this disaggregation based on the Merchant Classification Code (MCC) associated with each transaction. For each spending category, we select a basket of MCCs that are representative of spending in that category. We then analyze the spending in ZCTAs corresponding with the MCCs in each spending category. ${ }^{12}$

\footnotetext{
${ }^{12}$ For details about which MCCs are sorted into which spending categories, see the Appendix section C.
} 
Figure 2. Spending Categories with Economic Controls

Mar. 9 - Apr. 12

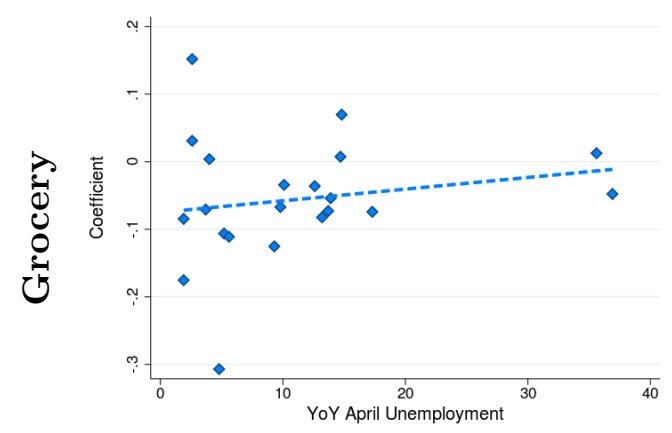

(a.i) Correlation: 0.182

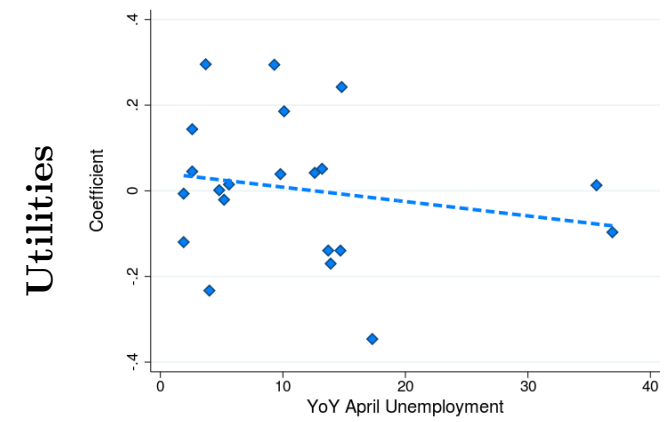

(b.i) Correlation: -0.194

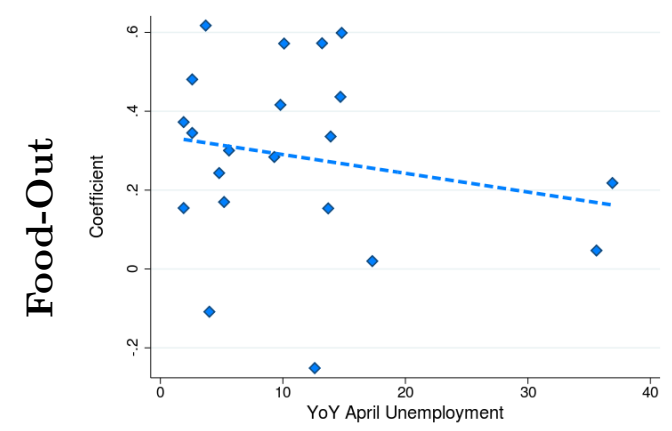

(c.i) Correlation: -0.198

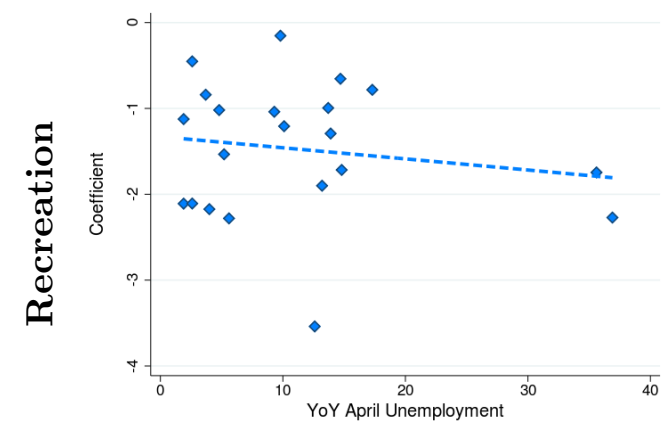

(d.i) Correlation: -0.160
Jul. 27 - Aug. 30
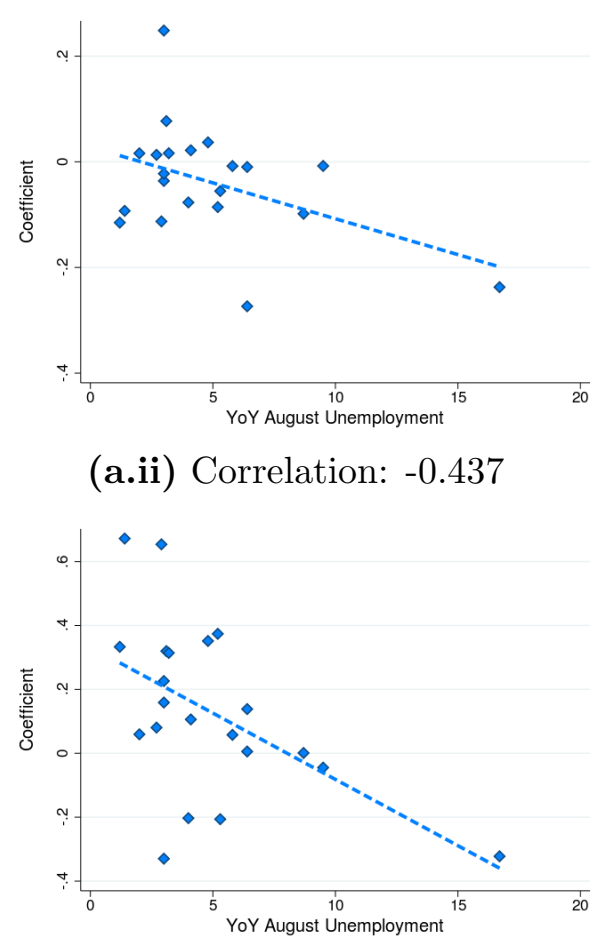

(b.ii) Correlation: -0.524

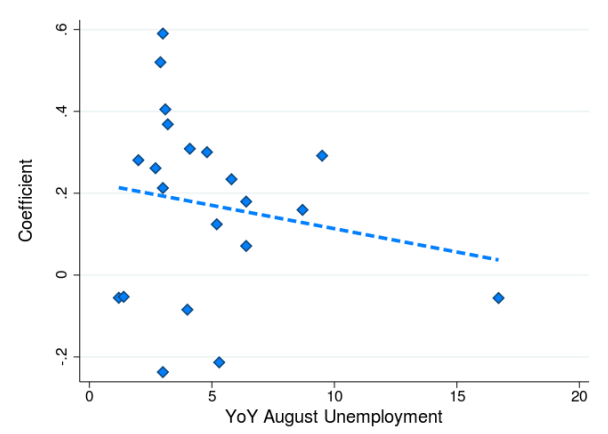

(c.ii) Correlation: -0.176

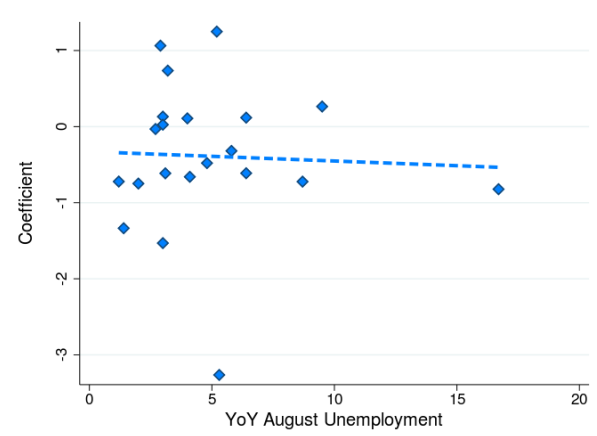

(d.ii) Correlation: -0.044

Sources: Unemployment figures from CPS, coefficients from authors' calculations using population data from the ACS and transaction data from Affinity.

We verify that similar patterns hold within spending categories. We apply a similar approach as 
before to study the relationship between occupational unemployment risk and spending in specific categories including economic controls. We apply the same regression structure as before but for spending by each spending category. ${ }^{13}$ We then plot the coefficients from these regressions for the pre-stimulus period and the post-stimulus period against the change in April and August yearover-year unemployment, respectively, in Figure 2. Rows represent spending on grocery, utilities, food-out, and recreation, respectively. Columns represent spending in the pre-stimulus and poststimulus periods, respectively.

We observe no consistent trend between unemployment risk and spending during the prestimulus period. Among the "essential" spending categories, we see a small positive relationship between grocery spending and unemployment risk (Figure 2[a.i]), whereas we observe a small negative relationship for utility spending (Figure 2[b.i]). Neither of these correlations is very far from zero, which seems to imply that during this early time period, unemployment risk had a small effect on essential spending. In the "nonessential" spending categories, food-out and recreation, we see small negative relationships between spending and unemployment risk (Figure 2[c.i] and Figure 2[d.i]). Again, as these relationships appear fairly weak, they seem to imply that during this early time period unemployment risk had little impact on nonessential spending.

We observe a negative relationship between all spending categories and unemployment risk during the post-stimulus period. The relationship between occupational unemployment risk and spending in the given categories is shown in subfigures a.ii, b.ii, c.ii, d.ii of Figure 2. All of these graphs show a negative relationship. This implies that unemployment risk had a largely negative impact on spending across both essential and nonessential goods categories. Note that this story mirrors what we saw when looking at overall spending. During the pre-stimulus period, unemployment risk had a non-negative impact on overall spending, possibly because workers in high-unemployment-risk occupations were anticipating future stimulus payments and benefited from expanded unemployment insurance. Once these benefits were wound down in the poststimulus period, unemployment risk had a negative relationship with overall spending as workers in high-unemployment-risk occupations were forced to cut spending relatively more.

\section{Characteristics of Unemployment Risk}

We explore a new measure of the degree to which workers in different occupations are exposed to unemployment risk. We have so far proxied for occupational unemployment risk using its realized unemployment rate. In this section, we look more closely at the characteristics of occupations that account for these differences in unemployment rates.

We build on other papers that study unemployment risk across occupations. We follow a similar method as Dingel and Neiman (2020), Mongey, Pilossoph, and Weinberg (2020), Montenovo et al. (2020), and Leibovici, Santacreu, and Famiglietti (2020) by using the O*NET Work Context and Work Activities surveys to construct an occupational unemployment risk measure. These authors use this data to create two main scores to measure how risky an occupation is: a work from home score and a proximity score.

The key novelty in our measure is that we introduce an additional measure of the degree to

\footnotetext{
${ }^{13}$ Results are given in the Appendix Section A.5.
} 
which jobs are considered essential. We approximate society's need for an occupation by looking at the occupation's "consequence of error" in the O*NET survey, which captures how serious it would be if a member of that occupation made a mistake that was not easily correctable. Therefore, we consider workers in an occupation to be at risk of unemployment if the occupation involves proximity to other people, cannot easily be performed at home, and has a low consequence of error.

Table 2. Occupational Risk Scores

\begin{tabular}{|c|c|c|c|c|c|c|c|}
\hline SOC Code & $\begin{array}{c}\text { YoY } \\
\text { Unemp. } \\
\text { April }\end{array}$ & $\begin{array}{c}\text { YoY } \\
\text { Unemp. } \\
\text { August }\end{array}$ & $\begin{array}{c}\text { Low } \\
\text { Cons. of } \\
\text { Error } \\
\text { Score }\end{array}$ & $\begin{array}{l}\text { Cannot } \\
\text { Work } \\
\text { from } \\
\text { Home } \\
\text { Score }\end{array}$ & $\begin{array}{l}\text { High } \\
\text { Prox. } \\
\text { Score }\end{array}$ & $\begin{array}{c}\text { High } \\
\text { Risk, All } \\
\text { Three } \\
\text { Scores }\end{array}$ & $\begin{array}{l}\text { High } \\
\text { Risk, } \\
\text { Exclude } \\
\text { Cons. } \\
\text { Score }\end{array}$ \\
\hline Food Preparation and Serving Related Occupations & 36.9 & 16.7 & .97 & .43 & .76 & Yes & Yes \\
\hline Personal Care and Service Occupations & 35.6 & 9.5 & .66 & .29 & .81 & Yes & Yes \\
\hline Building and Grounds Cleaning and Maintenance Occupations & 17.3 & 6.4 & 1 & .71 & .31 & No & No \\
\hline Production Occupations & 14.8 & 5.8 & .47 & .71 & .39 & No & No \\
\hline Arts, Design, Entertainment, Sports, and Media Occupations & 14.7 & 8.7 & .86 & 0 & .38 & No & No \\
\hline Transportation and Material Moving Occupations & 13.9 & 6.4 & .54 & .71 & .48 & No & Yes \\
\hline Construction and Extraction Occupations & 13.7 & 4 & .44 & 1 & .67 & No & Yes \\
\hline Sales and Related Occupations & 13.2 & 4.8 & .89 & .14 & .54 & No & No \\
\hline Educational Instruction, and Library Occupations & 12.6 & 5.3 & .88 & 0 & .62 & No & No \\
\hline Installation, Maintenance, and Repair Occupations & 10.1 & 4.1 & .45 & 1 & .51 & No & Yes \\
\hline Healthcare Support Occupations & 9.8 & 5.2 & .34 & .86 & 1 & No & Yes \\
\hline Office and Administrative Support Occupations & 9.3 & 3.2 & .9 & 0 & .36 & No & No \\
\hline Protective Service Occupations & 6.9 & 2.5 & .19 & .57 & .69 & No & Yes \\
\hline Healthcare Practitioners and Technical Occupations & 5.6 & 2 & 0 & .29 & .99 & No & Yes \\
\hline Management Occupations & 5.2 & 2.7 & .58 & 0 & .25 & No & No \\
\hline Life, Physical, and Social Science Occupations & 4.8 & 1.2 & .61 & 0 & .25 & No & No \\
\hline Business and Financial Operations Occupations & 4 & 3 & .75 & 0 & .16 & No & No \\
\hline Architecture and Engineering Occupations & 3.7 & 2.9 & .57 & 0 & .23 & No & No \\
\hline Legal Occupations & 2.6 & 3 & .23 & 0 & 0 & No & No \\
\hline Community and Social Service Occupations & 2.6 & 1.4 & .61 & .14 & .54 & No & No \\
\hline Computer and Mathematical Occupations & 1.9 & 3.1 & .58 & 0 & .29 & No & No \\
\hline Farming, Fishing, and Forestry Occupations & 1.9 & 3 & .58 & .57 & .04 & No & No \\
\hline
\end{tabular}

Source: Unemployment figures from CPS, occupational risk scores derived from O*NET data.

We follow Mongey, Pilossoph, and Weinberg (2020) and assign scores to each occupation along these dimensions and normalize the scores between 0 and $1 .^{14}$ Columns 4,5 and 6 of Table 2 display an occupation's normalized score in terms of "low consequence of error," "cannot work from home," and "proximity." Higher scores on these measures mean that a mistake on the job has less consequence, the occupation cannot be performed at home, and the occupation requires close proximity to others, respectively. We then combine these three scores to create a binary categorization of jobs as being "high risk" or not (shown in column 7 of Table 2). Occupations with an above-average median score in all three categories are categorized as high risk. Finally, to allow us to compare these scores with existing measures, we also compute a corresponding "high risk" measure based only on the degree to which an occupation cannot be performed at home and requires proximity to others. Under this alternative measure, occupations with an above-average median score in only these two categories are categorized as high risk.

Our measure appears to perform better than existing measures at predicting unemployment rates. Columns 2 and 3 show the change in unemployment in April 2020 and August 2020 relative to April 2019 and August 2019, respectively. The two "high risk" occupations according to our measure are occupations related to food preparation and serving and personal care and service occupations. Both of these occupation categories had more than 35 percent unemployment in April 2020; the next-highest occupation-category unemployment rate was 17.3 percent. They also

\footnotetext{
${ }^{14}$ For more information on how these scores are created, see the Appendix Section D.
} 
had the highest unemployment rates in August. The alternative measure indicates that protective services occupations and health-care practitioners and technical occupations were at high risk of unemployment, when in fact these occupations experienced relatively little unemployment in both April and August 2020. Therefore, we conclude that a key characteristic determining if workers in an occupation faced unemployment risk during the COVID-19 pandemic was whether that occupation is essential, in addition to the two previously discussed characteristics - whether it can be performed from home and whether it requires physical proximity to others on the job.

\section{Conclusion}

Policy interventions enacted in response to the spread of COVID-19 differentially impacted occupations in the first part of the pandemic, and as a result in workers in certain occupations faced significantly increased unemployment risk while workers in other occupations did not. Using a novel data set of high-frequency consumption spending, we investigate whether these differences in unemployment risk fed through to consumption spending. In particular, we analyze if those who were employed in risky occupations engaged in precautionary spending cuts. We find no evidence of precautionary cuts in the earlier part of the pandemic, but we find suggestive evidence that unemployment risk drove lower consumption spending as the pandemic proceeded, especially as the stimulus payments were phased out. Additionally, we create a new measure of occupational unemployment risk during the pandemic, which accurately predicts the worst affected occupations in terms of the realized unemployment rates.

An important aspect of the current analysis that could be improved by future research is the role of the CARES Act in governing spending patterns during the pandemic. While the results from both the pre-stimulus and post-stimulus periods can be explained by the timing of the act, a more formal investigation is required to establish any causal relationships. We plan to address this by supplementing our analysis with pre-paid card data that contain information on income receipts. 


\section{References}

Baker, Scott R., R. A. Farrokhnia, Steffen Meyer, Michaela Pagel, and Constantine Yannelis. 2020a. "How Does Household Spending Respond to an Epidemic? Consumption during the 2020 COVID-19 Pandemic." Working Paper 26949. National Bureau of Economic Research. doi:10.3386/w26949. Available at http://www.nber.org/papers/w26949.

Baker, Scott R., R. A. Farrokhnia, Steffen Meyer, Michaela Pagel, and Constantine Yannelis. 2020b. "Income, Liquidity, and the Consumption Response to the 2020 Economic Stimulus Payments." Working Paper 27097. National Bureau of Economic Research. doi:10.3386/w27097.

Available at http://www.nber.org/papers/w27097.

Basten, Christoph, Andreas Fagereng, and Kjetil Telle. 2016. "Saving and Portfolio Allocation before and after Job Loss." Journal of Money, Credit and Banking 48(2-3): 293-324.

Casado, Miguel Garza, Britta Glennon, Julia Lane, David McQuown, Daniel Rich, and Bruce A. Weinberg. 2020. "The Effect of Fiscal Stimulus: Evidence from COVID-19." Working Paper 27576. National Bureau of Economic Research. doi:10.3386/w27576.

Available at http://www.nber.org/papers/w27576.

Coibion, Olivier, Yuriy Gorodnichenko, and Michael Weber. 2020. "The Cost of the Covid-19 Crisis: Lockdowns, Macroeconomic Expectations, and Consumer Spending." Working Paper 27141. National Bureau of Economic Research. doi:10.3386/w27141.

Available at http://www.nber.org/papers/w27141.

Dingel, Jonathan I., and Brent Neiman. 2020. "How Many Jobs Can Be Done at Home?" Working Paper 26948. National Bureau of Economic Research. doi:10.3386/w26948.

Available at http://www.nber.org/papers/w26948.

Ganong, Peter, Pascal J. Noel, and Joseph S. Vavra. 2020. "US Unemployment Insurance Replacement Rates during the Pandemic." Working Paper 27216. National Bureau of Economic Research. doi: $10.3386 / \mathrm{w} 27216$.

Available at http://www.nber.org/papers/w27216.

Hendren, Nathan. 2017. "Knowledge of Future Job Loss and Implications for Unemployment Insurance." American Economic Review 107.

Karahan, Fatih, Brendan Moore, and Laura Pilossoph. 2019. "Expecting the Unexpected: Job Losses and Household Spending." Libery street economics blog. Federal Reserve Bank of New York.

Kumar, Raynil, and Shaun O’Brien. 2019. "2019 Findings from the Diary of Consumer Payment Choice.” Fednotes. Federal Reserve Bank of San Francisco.

Leibovici, Fernando, Ana Maria Santacreu, and Matthew Famiglietti. 2020. "Social Distancing and Contact-Intensive Occupations." On the economy blog. St. Louis Federal Reserve.

Mongey, Simon, Laura Pilossoph, and Alex Weinberg. 2020. "Which Workers Bear the Burden of Social Distancing Policies?" Working Paper 27085. National Bureau of Economic Research. doi:10.3386/ w27085.

Available at http://www.nber.org/papers/w27085. 
Montenovo, Laura, Xuan Jiang, Felipe Lozano Rojas, Ian M. Schmutte, Kosali I. Simon, Bruce A. Weinberg, and Coady Wing. 2020. "Determinants of Disparities in Covid-19 Job Losses." Working Paper 27132. National Bureau of Economic Research. doi:10.3386/w27132.

Available at http://www.nber.org/papers/w27132.

Skinner, Jonathan. 1988. "Risky Income, Life Cycle Consumption, and Precautionary Savings." Journal of Monetary Economics 22: 237-255.

Stephens, Melvin Jr. 2004. "Job Loss Expectations, Realizations, and Household Consumption Behavior." The Review of Economics and Statistics 86: 253-269. 


\section{Appendices}

\section{A Additional Results}

\section{A.1 Controls}

Table 3 explores the relationship between occupation and consumption spending as our controls are introduced. Regression (1) shows the estimates from regressing log consumer spending on the share of occupations by Zip Code Tabulation Area (ZCTA) and state fixed effects. ${ }^{15}$ To allow for common changes in spending across ZCTAs, we include weekly dummies in regression (2), which do not affect the results significantly. Next, we control for common changes in lockdown policies across states in regression (3) by including post-COVID state interaction terms. Again, these don't change the coefficients substantially. Finally, we introduce interactions between demographic variables and whether or not the week is post-COVID in regression (4). The coefficients for these interaction terms are given in regression (1) of Table 6.

Table 3. Basic Regressions (3/9/2020-4/12/2020)

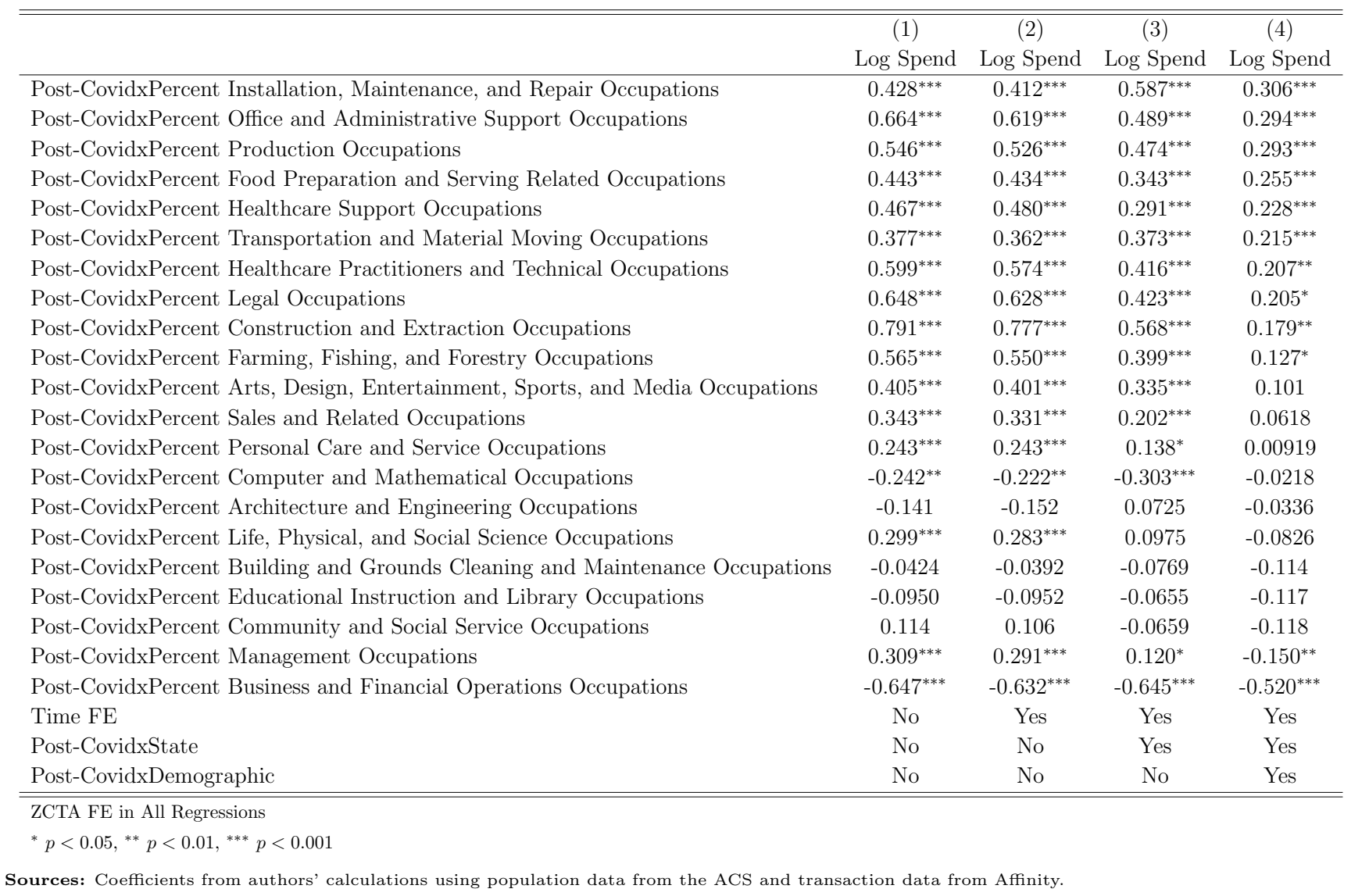

\footnotetext{
${ }^{15}$ We also include a dummy for whether or not the time period is in the post-COVID period in regression (1) to allow a standard difference-in-difference interpretation.
} 


\section{A.2 All Five-week Time Periods}

As discussed in Section 3, Table 4 and Table 5 show the standard regression results for five consecutive five-week periods without and with economic controls, respectively. These results are summarized graphically in Figure 1. Note that regression (1) of Table 4 is the same as regression (4) of Table 3, and more detailed tables for some of these regressions are available in Table 6.

Table 4. Regression Results Separated by Five-week Time Periods without Economic Controls

\begin{tabular}{|c|c|c|c|c|c|}
\hline & $\begin{array}{c}3 / 9-4 / 12 \\
(1) \\
\log \text { Spend }\end{array}$ & $\begin{array}{c}4 / 13-5 / 17 \\
(2) \\
\log \text { Spend }\end{array}$ & $\begin{array}{c}5 / 18-6 / 22 \\
(3) \\
\log \text { Spend }\end{array}$ & $\begin{array}{c}6 / 23-7 / 26 \\
(4) \\
\log \text { Spend }\end{array}$ & $\begin{array}{c}7 / 27-8 / 30 \\
(5) \\
\text { Log Spend }\end{array}$ \\
\hline Post-CovidxPercent Installation, Maintenance, and Repair Occupations & $0.306^{* * *}$ & $0.360^{* * *}$ & 0.101 & $0.280^{* * *}$ & $0.222^{* * *}$ \\
\hline Post-CovidxPercent Office and Administrative Support Occupations & $0.294^{* * *}$ & $0.211^{* * *}$ & $0.139^{*}$ & $0.263^{* * *}$ & $0.276^{* * *}$ \\
\hline Post-CovidxPercent Production Occupations & $0.293^{* * *}$ & $0.279^{* * *}$ & -0.0881 & $0.158^{*}$ & 0.0413 \\
\hline Post-CovidxPercent Food Preparation and Serving Related Occupations & $0.255^{* * *}$ & 0.0621 & -0.0712 & 0.00592 & $-0.133^{*}$ \\
\hline Post-CovidxPercent Healthcare Support Occupations & $0.228^{* * *}$ & $0.312^{* * *}$ & 0.0800 & $0.180^{* *}$ & 0.0487 \\
\hline Post-CovidxPercent Transportation and Material Moving Occupations & $0.215^{* * *}$ & $0.148^{*}$ & -0.0674 & $0.164^{* *}$ & $0.127^{*}$ \\
\hline Post-CovidxPercent Healthcare Practitioners and Technical Occupations & $0.207^{* *}$ & 0.0903 & -0.135 & $0.218^{* *}$ & $0.225^{* *}$ \\
\hline Post-CovidxPercent Legal Occupations & $0.205^{*}$ & 0.104 & -0.135 & 0.0617 & 0.0962 \\
\hline Post-CovidxPercent Construction and Extraction Occupations & $0.179^{* *}$ & 0.121 & -0.0931 & 0.0374 & -0.0180 \\
\hline Post-CovidxPercent Farming, Fishing, and Forestry Occupations & $0.127^{*}$ & $0.259^{* * *}$ & 0.0629 & $0.198^{* * *}$ & $0.230^{* * *}$ \\
\hline Post-CovidxPercent Arts, Design, Entertainment, Sports, and Media Occupations & 0.101 & 0.0403 & 0.0914 & $0.170^{* *}$ & 0.0777 \\
\hline Post-CovidxPercent Sales and Related Occupations & 0.0618 & $0.246^{* * *}$ & 0.0308 & $0.255^{* * *}$ & $0.225^{* * *}$ \\
\hline Post-CovidxPercent Personal Care and Service Occupations & 0.00919 & 0.000808 & $-0.147^{*}$ & 0.0695 & $0.136^{*}$ \\
\hline Post-CovidxPercent Computer and Mathematical Occupations & -0.0218 & -0.110 & -0.115 & -0.00281 & 0.00908 \\
\hline Post-CovidxPercent Architecture and Engineering Occupations & -0.0336 & -0.0593 & 0.00105 & -0.0667 & $0.284^{* *}$ \\
\hline Post-CovidxPercent Life, Physical, and Social Science Occupations & -0.0826 & -0.0436 & $-0.252^{* * *}$ & -0.0266 & 0.0177 \\
\hline Post-CovidxPercent Building and Grounds Cleaning and Maintenance Occupations & -0.114 & $-0.200^{*}$ & $-0.201^{*}$ & -0.123 & -0.0900 \\
\hline Post-CovidxPercent Educational Instruction and Library Occupations & -0.117 & $-0.220^{*}$ & $-0.421^{* * *}$ & $-0.275^{* *}$ & -0.114 \\
\hline Post-CovidxPercent Community and Social Service Occupations & -0.118 & -0.194 & $-0.323^{*}$ & 0.131 & -0.0531 \\
\hline Post-CovidxPercent Management Occupations & $-0.150^{* *}$ & $-0.215^{* * *}$ & $-0.295^{* * *}$ & 0.00367 & -0.0409 \\
\hline Post-CovidxPercent Business and Financial Operations Occupations & $-0.520^{* * *}$ & $-0.662^{* * *}$ & $-0.821^{* * *}$ & $-0.433^{* * *}$ & $-0.503^{* * *}$ \\
\hline
\end{tabular}

Sources: Coefficients from authors' calculations using population data from the ACS and transaction data from Affinity.

Table 5. Regression Results Separated by Five-week Time Periods with Economic Controls

\begin{tabular}{|c|c|c|c|c|c|}
\hline & $\begin{array}{c}3 / 9-4 / 12 \\
(1) \\
\text { Log Spend }\end{array}$ & $\begin{array}{c}/ 13-5 / 17 \\
(2) \\
\text { Log Spend }\end{array}$ & $\begin{array}{c}5 / 18-6 / 22 \\
(3) \\
\text { Log Spend }\end{array}$ & $\begin{array}{c}6 / 23-7 / 26 \\
(4) \\
\text { Log Spend }\end{array}$ & $\begin{array}{c}7 / 27-8 / 30 \\
(5) \\
\text { Log Spend }\end{array}$ \\
\hline Post-CovidxPercent Office and Administrative Support Occupations & $0.329^{* * *}$ & $0.192^{* *}$ & $0.214^{* *}$ & $0.276^{* * *}$ & $0.301^{* * *}$ \\
\hline Post-CovidxPercent Computer and Mathematical Occupations & $0.302^{* *}$ & $0.330^{* *}$ & $0.386^{* * *}$ & $0.471^{* * *}$ & $0.390^{* * *}$ \\
\hline Post-CovidxPercent Production Occupations & $0.278^{* * *}$ & $0.163^{*}$ & -0.101 & 0.112 & 0.0242 \\
\hline Post-CovidxPercent Installation, Maintenance, and Repair Occupations & $0.272^{* * *}$ & $0.187^{* *}$ & 0.0747 & $0.155^{*}$ & $0.171^{* *}$ \\
\hline Post-CovidxPercent Food Preparation and Serving Related Occupations & $0.247^{* * *}$ & -0.00910 & -0.0650 & -0.0246 & -0.109 \\
\hline Post-CovidxPercent Transportation and Material Moving Occupations & $0.230^{* * *}$ & 0.120 & 0.00514 & $0.180^{* *}$ & $0.161^{*}$ \\
\hline Post-CovidxPercent Architecture and Engineering Occupations & $0.218^{*}$ & 0.183 & $0.227^{*}$ & 0.194 & $0.386^{* * *}$ \\
\hline Post-CovidxPercent Legal Occupations & $0.211^{*}$ & 0.164 & 0.0809 & $0.203^{*}$ & 0.156 \\
\hline Post-CovidxPercent Arts, Design, Entertainment, Sports, and Media Occupations & $0.190^{* *}$ & 0.0583 & $0.205^{* *}$ & $0.186^{* *}$ & 0.0934 \\
\hline Post-CovidxPercent Healthcare Practitioners and Technical Occupations & $0.183^{*}$ & 0.0360 & 0.00534 & $0.217^{*}$ & $0.285^{* *}$ \\
\hline Post-CovidxPercent Healthcare Support Occupations & $0.171^{*}$ & $0.243^{* * *}$ & 0.0632 & $0.167^{*}$ & 0.0543 \\
\hline Post-CovidxPercent Farming, Fishing, and Forestry Occupations & $0.150^{*}$ & $0.188^{* *}$ & $0.138^{*}$ & $0.176^{* *}$ & $0.254^{* * *}$ \\
\hline Post-CovidxPercent Life, Physical, and Social Science Occupations & 0.117 & 0.132 & -0.130 & 0.0219 & 0.108 \\
\hline Post-CovidxPercent Construction and Extraction Occupations & 0.108 & -0.0262 & $-0.151^{*}$ & -0.0442 & 0.00545 \\
\hline Post-CovidxPercent Personal Care and Service Occupations & 0.0931 & 0.0522 & -0.0163 & $0.151^{*}$ & $0.243^{* * *}$ \\
\hline Post-CovidxPercent Sales and Related Occupations & 0.0887 & $0.243^{* * *}$ & $0.153^{*}$ & $0.294^{* * *}$ & $0.322^{* * *}$ \\
\hline Post-CovidxPercent Educational Instruction and Library Occupations & -0.0219 & -0.0574 & $-0.200^{*}$ & -0.187 & 0.0640 \\
\hline Post-CovidxPercent Management Occupations & -0.0287 & $-0.167^{* *}$ & $-0.184^{* *}$ & 0.0274 & 0.0497 \\
\hline Post-CovidxPercent Building and Grounds Cleaning and Maintenance Occupations & -0.0700 & -0.120 & -0.0915 & -0.131 & -0.122 \\
\hline Post-CovidxPercent Community and Social Service Occupations & -0.214 & $-0.318^{*}$ & -0.162 & 0.145 & -0.106 \\
\hline Post-CovidxPercent Business and Financial Operations Occupations & $-0.324^{* * *}$ & $-0.451^{* * *}$ & $-0.566^{* * *}$ & $-0.312^{* *}$ & $-0.345^{* * *}$ \\
\hline Post-CovidxLog Median Income & $-0.0148^{* *}$ & 0.00743 & 0.00381 & -0.00104 & 0.00293 \\
\hline Post-CovidxPercent age 25-plus with some college degree & $-0.157^{* * *}$ & $-0.271^{* * *}$ & $-0.207^{* * *}$ & $-0.167^{* * *}$ & $-0.139^{* * *}$ \\
\hline
\end{tabular}

Sources: Coefficients from authors' calculations using population data from the ACS and transaction data from Affinity. 


\section{A.3 Full Results}

Table 6 gives complete regression results including all economic and demographic controls and standard errors. These regressions still omit coefficients for state interaction terms and ZCTA/time fixed effect terms, as there would be more than can be easily shown here. Regressions (1) and (2) are identical to regression (1) in Table 4 and regression (1) in Table 5, respectively.

Table 6. Pre-stimulus Results, Showing All Controls

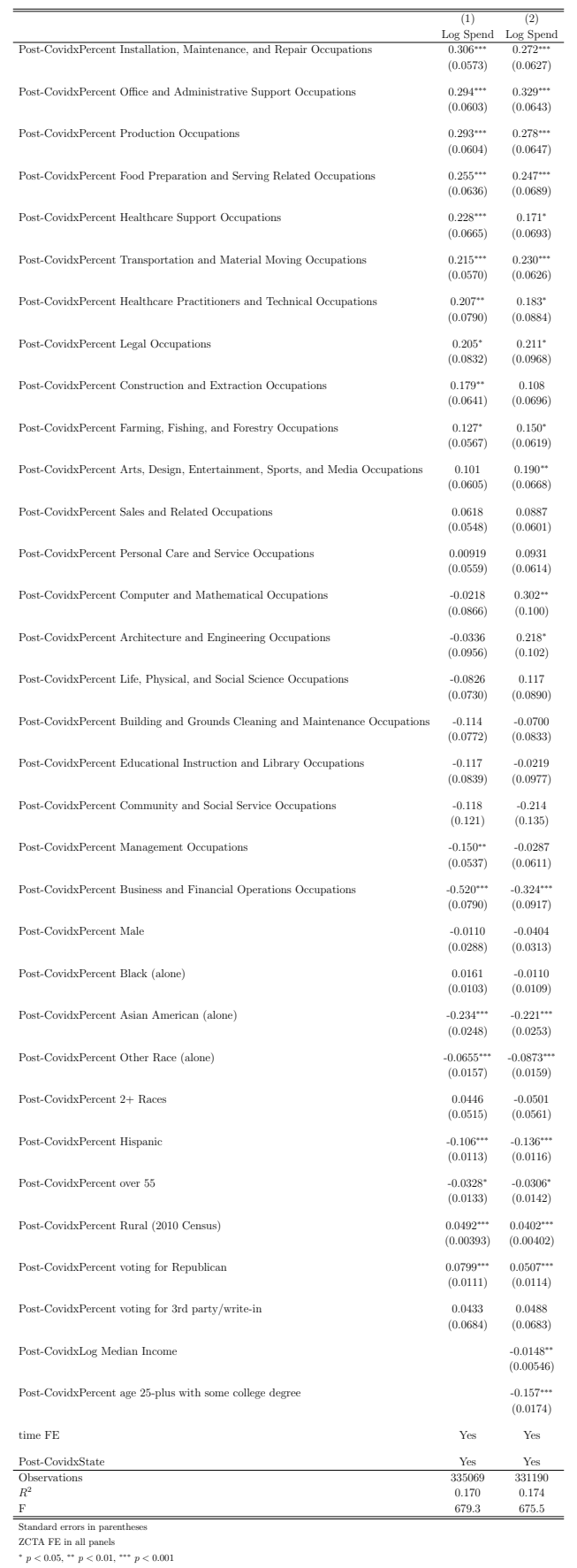

Sources: Coefficients from authors' calculations using population data from the ACS and transaction data from Affinity. 


\section{A.4 Pre-stimulus Spending and Long-term Unemployment Risk}

As discussed in Section 3.2, we verify that the relationship between occupational unemployment risk and pre-stimulus spending is unchanged with a measure of long-term unemployment risk. We therefore look at the relationship between our coefficients for consumption spending and the change in unemployment in occupations from August 2019 to August 2020 (these variables are given in Table 1). We can see that there were large falls in unemployment across occupations from April to August 2020. However, these falls were not constant across occupations. For example, while unemployment remained relatively high in food preparation occupations (36.9 percent to 16.7 percent), it fell relatively more in construction occupations (13.7 percent to 4.0 percent). In this case, construction occupation workers faced high short-term risk of unemployment but lower long-term risk. Using the same method as before, we graphically depict the relationship in Figure 3. Both with and without economic controls, a higher rise in unemployment in August is associated with relatively higher consumption spending during the pre-stimulus period.

Figure 3. Relation between Long-term Unemployment Risk and Relative Spending from 3/9/2020$4 / 12 / 2020$

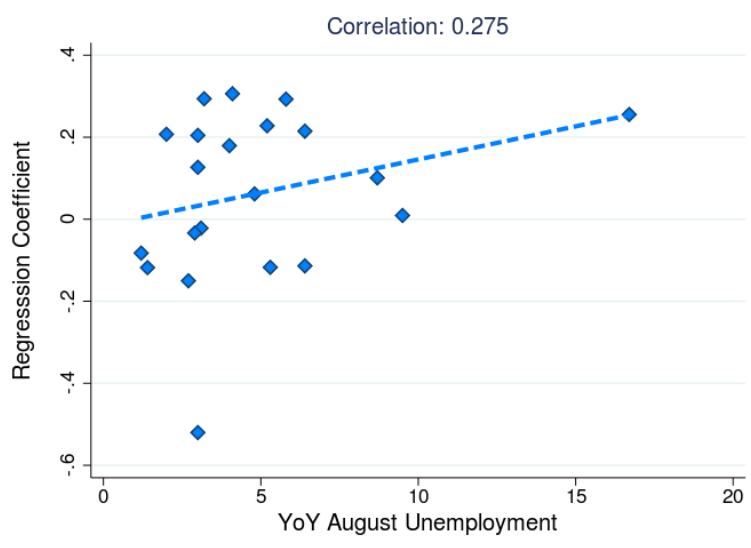

(a) No Economic Controls

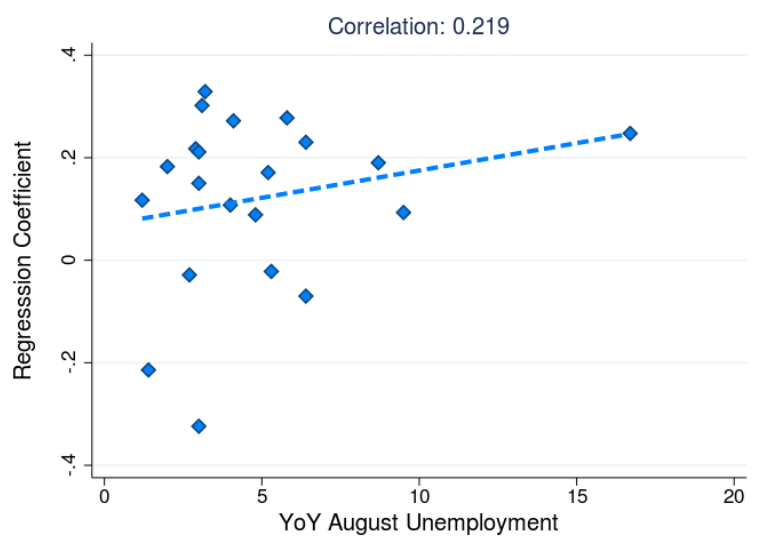

(b) With Economic Controls

Sources: Unemployment figures from CPS, coefficients from authors' calculations using population data from the ACS and transaction data from Affinity.

\section{A.5 Spending by Category Regression Results}

As mentioned in Section 3.5, Tables 7 and 8 show the standard regression with economic controls by spending category for the pre-stimulus and post-stimulus periods, respectively. 
Table 7. Spending by Category, Pre-stimulus

\begin{tabular}{|c|c|c|c|c|c|}
\hline & $\begin{array}{c}\text { All Categories } \\
\text { (1) } \\
\text { Log Spend }\end{array}$ & $\begin{array}{c}\text { Grocery } \\
(2) \\
\text { Log Spend }\end{array}$ & $\begin{array}{c}\text { Food-out } \\
(3) \\
\text { Log Spend }\end{array}$ & $\begin{array}{c}\text { Recreation } \\
\quad(4) \\
\text { Log Spend }\end{array}$ & $\begin{array}{c}\text { Utilities } \\
\text { (5) } \\
\text { Log Spend }\end{array}$ \\
\hline Post-CovidxPercent Office and Administrative Support Occupations & $0.329^{* * *}$ & $-0.125^{*}$ & $0.284^{* * *}$ & $-1.039^{*}$ & $0.294^{*}$ \\
\hline Post-CovidxPercent Computer and Mathematical Occupations & $0.302^{* *}$ & $-0.175^{*}$ & $0.373^{* *}$ & $-2.109^{* * *}$ & -0.00664 \\
\hline Post-CovidxPercent Production Occupations & $0.278^{* * *}$ & 0.0697 & $0.599^{* * *}$ & $-1.716^{* * *}$ & 0.242 \\
\hline Post-CovidxPercent Installation, Maintenance, and Repair Occupations & $0.272^{* * *}$ & -0.0343 & $0.572^{* * *}$ & $-1.207^{* *}$ & 0.186 \\
\hline Post-CovidxPercent Food Preparation and Serving Related Occupations & $0.247^{* * *}$ & -0.0478 & $0.218^{*}$ & $-2.271^{* * *}$ & -0.0967 \\
\hline Post-CovidxPercent Transportation and Material Moving Occupations & $0.230^{* * *}$ & -0.0540 & $0.336^{* * *}$ & $-1.292^{* *}$ & -0.170 \\
\hline Post-CovidxPercent Architecture and Engineering Occupations & $0.218^{*}$ & -0.0708 & $0.617^{* * *}$ & -0.840 & 0.296 \\
\hline Post-CovidxPercent Legal Occupations & $0.211^{*}$ & 0.152 & $0.481^{* * *}$ & -0.452 & 0.144 \\
\hline Post-CovidxPercent Arts, Design, Entertainment, Sports, and Media Occupations & $0.190^{* *}$ & 0.00739 & $0.437^{* * *}$ & -0.655 & -0.140 \\
\hline Post-CovidxPercent Healthcare Practitioners and Technical Occupations & $0.183^{*}$ & -0.111 & $0.300^{* *}$ & $-2.281^{* * *}$ & 0.0148 \\
\hline Post-CovidxPercent Healthcare Support Occupations & $0.171^{*}$ & -0.0671 & $0.416^{* * *}$ & -0.153 & 0.0390 \\
\hline Post-CovidxPercent Farming, Fishing, and Forestry Occupations & $0.150^{*}$ & -0.0846 & $0.155^{*}$ & $-1.124^{* *}$ & -0.120 \\
\hline Post-CovidxPercent Life, Physical, and Social Science Occupations & 0.117 & $-0.307^{* * *}$ & $0.243^{*}$ & -1.019 & 0.00149 \\
\hline Post-CovidxPercent Construction and Extraction Occupations & 0.108 & -0.0729 & 0.154 & -0.995 & -0.139 \\
\hline Post-CovidxPercent Personal Care and Service Occupations & 0.0931 & 0.0125 & 0.0469 & $-1.746^{* * *}$ & 0.0130 \\
\hline Post-CovidxPercent Sales and Related Occupations & 0.0887 & -0.0823 & $0.572^{* * *}$ & $-1.900^{* * *}$ & 0.0514 \\
\hline Post-CovidxPercent Educational Instruction and Library Occupations & -0.0219 & -0.0361 & $-0.251^{*}$ & $-3.540^{* * *}$ & 0.0420 \\
\hline Post-CovidxPercent Management Occupations & -0.0287 & $-0.106^{*}$ & $0.170^{*}$ & $-1.534^{* * *}$ & -0.0209 \\
\hline Post-CovidxPercent Building and Grounds Cleaning and Maintenance Occupations & -0.0700 & -0.0741 & 0.0199 & -0.783 & $-0.346^{*}$ \\
\hline Post-CovidxPercent Community and Social Service Occupations & -0.214 & 0.0309 & $0.345^{*}$ & $-2.108^{*}$ & 0.0452 \\
\hline Post-CovidxPercent Business and Financial Operations Occupations & $-0.324^{* * *}$ & 0.00381 & -0.108 & $-2.173^{* * *}$ & -0.233 \\
\hline Post-CovidxLog Median Income & $-0.0148^{* *}$ & 0.00874 & $-0.0275^{* * *}$ & $-0.129^{* * *}$ & -0.00499 \\
\hline Post-CovidxPercent age 25 -plus with some college degree & $-0.157^{* * *}$ & 0.0154 & $-0.327^{* * *}$ & -0.117 & 0.0326 \\
\hline
\end{tabular}

ZCTA FE, Time FE, Post-CovidxState interaction terms, and Post-CovidxDemographic interaction terms included in all panels

${ }^{*} p<0.05,{ }^{* *} p<0.01,{ }^{* * *} p<0.001$

Sources: Coefficients from authors' calculations using population data from the ACS and transaction data from Affinity.

Table 8. Spending by Category, Post-Stimulus

\begin{tabular}{|c|c|c|c|c|c|}
\hline & $\begin{array}{c}\text { All Categories } \\
\text { (1) } \\
\text { Log Spend }\end{array}$ & $\begin{array}{c}\text { Grocery } \\
(2) \\
\text { Log Spend }\end{array}$ & $\begin{array}{c}\text { Food-out } \\
(3) \\
\text { Log Spend }\end{array}$ & $\begin{array}{c}\text { Recreation } \\
\quad(4) \\
\text { Log Spend }\end{array}$ & $\begin{array}{c}\text { Utilities } \\
\quad(5) \\
\text { Log Spend }\end{array}$ \\
\hline Post-CovidxPercent Computer and Mathematical Occupations & $0.390^{* * *}$ & 0.0771 & $0.405^{* * *}$ & -0.614 & 0.320 \\
\hline Post-CovidxPercent Architecture and Engineering Occupations & $0.386^{* * *}$ & -0.113 & $0.520^{* * *}$ & 1.065 & $0.654^{* *}$ \\
\hline Post-CovidxPercent Sales and Related Occupations & $0.322^{* * *}$ & 0.0369 & $0.300^{* * *}$ & -0.479 & $0.351^{* *}$ \\
\hline Post-CovidxPercent Office and Administrative Support Occupations & $0.301^{* * *}$ & 0.0163 & $0.369^{* * *}$ & 0.736 & $0.314^{*}$ \\
\hline Post-CovidxPercent Healthcare Practitioners and Technical Occupations & $0.285^{* *}$ & 0.0161 & $0.281^{* *}$ & -0.748 & 0.0594 \\
\hline Post-CovidxPercent Farming, Fishing, and Forestry Occupations & $0.254^{* * *}$ & -0.0224 & $0.213^{* *}$ & 0.0259 & 0.226 \\
\hline Post-CovidxPercent Personal Care and Service Occupations & $0.243^{* * *}$ & -0.00780 & $0.292^{* * *}$ & 0.264 & -0.0448 \\
\hline Post-CovidxPercent Installation, Maintenance, and Repair Occupations & $0.171^{* *}$ & 0.0218 & $0.309^{* * *}$ & -0.660 & 0.106 \\
\hline Post-CovidxPercent Transportation and Material Moving Occupations & $0.161^{*}$ & -0.00979 & $0.180^{*}$ & 0.118 & 0.00551 \\
\hline Post-CovidxPercent Legal Occupations & 0.156 & $0.249^{* *}$ & $0.590^{* * *}$ & 0.131 & 0.159 \\
\hline Post-CovidxPercent Life, Physical, and Social Science Occupations & 0.108 & -0.115 & -0.0555 & -0.722 & 0.333 \\
\hline Post-CovidxPercent Arts, Design, Entertainment, Sports, and Media Occupations & 0.0934 & -0.0984 & 0.159 & -0.723 & 0.000979 \\
\hline Post-CovidxPercent Educational Instruction and Library Occupations & 0.0640 & -0.0554 & -0.213 & $-3.264^{* * *}$ & -0.206 \\
\hline Post-CovidxPercent Healthcare Support Occupations & 0.0543 & -0.0857 & 0.124 & $1.249^{*}$ & $0.374^{*}$ \\
\hline Post-CovidxPercent Management Occupations & 0.0497 & 0.0132 & $0.261^{* * *}$ & -0.0313 & 0.0804 \\
\hline Post-CovidxPercent Production Occupations & 0.0242 & -0.00799 & $0.234^{* *}$ & -0.319 & 0.0577 \\
\hline Post-CovidxPercent Construction and Extraction Occupations & 0.00545 & -0.0769 & -0.0845 & 0.109 & -0.203 \\
\hline Post-CovidxPercent Community and Social Service Occupations & -0.106 & -0.0928 & -0.0531 & -1.336 & $0.672^{*}$ \\
\hline Post-CovidxPercent Food Preparation and Serving Related Occupations & -0.109 & $-0.237^{* * *}$ & -0.0561 & -0.822 & $-0.322^{*}$ \\
\hline Post-CovidxPercent Building and Grounds Cleaning and Maintenance Occupations & -0.122 & $-0.274^{* * *}$ & 0.0711 & -0.612 & 0.138 \\
\hline Post-CovidxPercent Business and Financial Operations Occupations & $-0.345^{* * *}$ & -0.0361 & $-0.237^{*}$ & $-1.531^{* *}$ & -0.330 \\
\hline Post-CovidxLog Median Income & 0.00293 & 0.00103 & $0.0274^{* * *}$ & $-0.159^{* * *}$ & $-0.0267^{*}$ \\
\hline Post-CovidxPercent age 25-plus with some college degree & $-0.139^{* * *}$ & 0.0297 & $-0.376^{* * *}$ & $-0.240^{*}$ & $-0.0958^{* *}$ \\
\hline
\end{tabular}

Sources: Coefficients from authors' calculations using population data from the ACS and transaction data from Affinity.

\section{B Robustness Checks}

We conduct several robustness checks to verify the validity of our approach.

\section{B.1 Falsification Test}

To verify that the empirical approach is not picking up on spurious correlations between occupations and consumption spending, we conduct a falsification test. We replicate regression (1) 
in Table 5 for the same weeks in 2019 rather than 2020. The results are given in Table 9. Regression (1) shows the results for 2020, while regression (2) shows the results for 2019. Unlike in 2020, occupation explains very little of the change in consumption spending across ZCTAs in 2019. This suggests that we are not picking up on spurious correlations and that the pandemic caused significant differences in consumption spending between workers in different occupations.

Table 9. Falsification Test: Comparing Regression Results from 2019 with 2020

\begin{tabular}{|c|c|c|}
\hline & $\begin{array}{c}\text { 3/9/20-4/12/20 } \\
(1) \\
\text { Log Spend }\end{array}$ & $\begin{array}{c}\text { 3/9/19-4/12/19 } \\
(2) \\
\text { Log Spend }\end{array}$ \\
\hline Post-CovidxPercent Office and Administrative Support Occupations & $0.329^{* * *}$ & $-0.212^{* * *}$ \\
\hline Post-CovidxPercent Computer and Mathematical Occupations & $0.302^{* *}$ & -0.132 \\
\hline Post-CovidxPercent Production Occupations & $0.278^{* * *}$ & -0.0661 \\
\hline Post-CovidxPercent Installation, Maintenance, and Repair Occupations & $0.272^{* * *}$ & -0.0402 \\
\hline Post-CovidxPercent Food Preparation and Serving Related Occupations & $0.247^{* * *}$ & -0.0421 \\
\hline Post-CovidxPercent Transportation and Material Moving Occupations & $0.230^{* * *}$ & -0.0879 \\
\hline Post-CovidxPercent Architecture and Engineering Occupations & $0.218^{*}$ & 0.0451 \\
\hline Post-CovidxPercent Legal Occupations & $0.211^{*}$ & 0.0370 \\
\hline Post-CovidxPercent Arts, Design, Entertainment, Sports, and Media Occupations & $0.190^{* *}$ & -0.105 \\
\hline Post-CovidxPercent Healthcare Practitioners and Technical Occupations & $0.183^{*}$ & -0.125 \\
\hline Post-CovidxPercent Healthcare Support Occupations & $0.171^{*}$ & 0.0813 \\
\hline Post-CovidxPercent Farming, Fishing, and Forestry Occupations & $0.150^{*}$ & -0.0430 \\
\hline Post-CovidxPercent Life, Physical, and Social Science Occupations & 0.117 & -0.105 \\
\hline Post-CovidxPercent Construction and Extraction Occupations & 0.108 & -0.0263 \\
\hline Post-CovidxPercent Personal Care and Service Occupations & 0.0931 & -0.0885 \\
\hline Post-CovidxPercent Sales and Related Occupations & 0.0887 & -0.0578 \\
\hline Post-CovidxPercent Educational Instruction and Library Occupations & -0.0219 & -0.0659 \\
\hline Post-CovidxPercent Management Occupations & -0.0287 & -0.0682 \\
\hline Post-CovidxPercent Building and Grounds Cleaning and Maintenance Occupations & -0.0700 & -0.0691 \\
\hline Post-CovidxPercent Community and Social Service Occupations & -0.214 & 0.00697 \\
\hline Post-CovidxPercent Business and Financial Operations Occupations & $-0.324^{* * *}$ & -0.0909 \\
\hline Post-CovidxLog Median Income & $-0.0148^{* *}$ & -0.00874 \\
\hline Post-CovidxPercent age 25-plus with some college degree & $-0.157^{* * *}$ & -0.0129 \\
\hline
\end{tabular}

Sources: Coefficients from authors' calculations using population data from the ACS and transaction data from Affinity.

\section{B.2 Controlling for Variation in Spending between ZCTAs}

Another concern is that the impact of a worker's occupation on their post-COVID spending is being influenced disproportionately by specific ZCTAs. Our empirical approach is to analyze log change in consumption spending across ZCTAs. This means that a small ZCTA, which could have large proportional changes across periods, is weighted equally with a large ZCTA. A histogram of total weekly log spending by ZCTA is displayed in Figure 4. We see that the histogram does not have any significant outliers. In order to be sure that values at either end of this distribution are not driving the relationship that we find between occupation and spending, we drop observations in the top and bottom 10th percentiles of this distribution and then replicate regression (1) in Table $5 .{ }^{16}$ The results of this regression are shown in Table 10. Regression (1) shows the original regression, while regression (2) shows the coefficients with the dropped points. We see very similar coefficients with and without dropping the ends of the distribution, suggesting that outliers are not driving our results.

\footnotetext{
${ }^{16}$ We are more concerned about the bottom 10 percent driving the results but exclude both the bottom 10 percent and top 1 percent out of a desire for symmetric changes to both ends of the distribution.
} 
Figure 4. Histogram of Log Spending by ZCTA

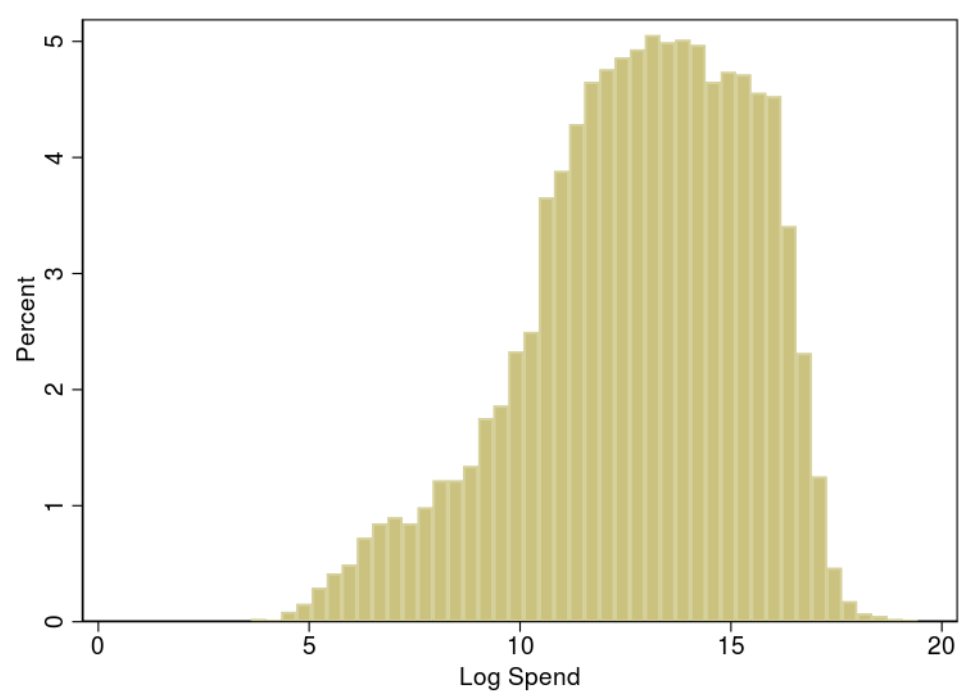

Source: Affinity.

Table 10. Pre-stimulus Results, Removing Top and Bottom $10 \%$ by Spending

\begin{tabular}{|c|c|c|}
\hline & $\begin{array}{c}\text { All Obervations } \\
\text { (1) } \\
\text { Log Spend }\end{array}$ & $\begin{array}{l}\text { Remove Top and Bottom 10th Percentiles } \\
\text { (2) } \\
\text { Log Spend }\end{array}$ \\
\hline Post-CovidxPercent Office and Administrative Support Occupations & $0.329^{* * *}$ & $0.314^{* * *}$ \\
\hline Post-CovidxPercent Computer and Mathematical Occupations & $0.302^{* *}$ & $0.327^{* *}$ \\
\hline Post-CovidxPercent Production Occupations & $0.278^{* * *}$ & $0.292^{* * *}$ \\
\hline Post-CovidxPercent Installation, Maintenance, and Repair Occupations & $0.272^{* * *}$ & $0.298^{* * *}$ \\
\hline Post-CovidxPercent Food Preparation and Serving Related Occupations & $0.247^{* * *}$ & $0.262^{* * *}$ \\
\hline Post-CovidxPercent Transportation and Material Moving Occupations & $0.230^{* * *}$ & $0.225^{* * *}$ \\
\hline Post-CovidxPercent Architecture and Engineering Occupations & $0.218^{*}$ & $0.222^{*}$ \\
\hline Post-CovidxPercent Legal Occupations & $0.211^{*}$ & 0.195 \\
\hline Post-CovidxPercent Arts, Design, Entertainment, Sports, and Media Occupations & $0.190^{* *}$ & $0.191^{* *}$ \\
\hline Post-CovidxPercent Healthcare Practitioners and Technical Occupations & $0.183^{*}$ & 0.118 \\
\hline Post-CovidxPercent Healthcare Support Occupations & $0.171^{*}$ & $0.197^{* *}$ \\
\hline Post-CovidxPercent Farming, Fishing, and Forestry Occupations & $0.150^{*}$ & $0.160^{*}$ \\
\hline Post-CovidxPercent Life, Physical, and Social Science Occupations & 0.117 & 0.0992 \\
\hline Post-CovidxPercent Construction and Extraction Occupations & 0.108 & 0.0857 \\
\hline Post-CovidxPercent Personal Care and Service Occupations & 0.0931 & 0.0838 \\
\hline Post-CovidxPercent Sales and Related Occupations & 0.0887 & 0.123 \\
\hline Post-CovidxPercent Educational Instruction and Library Occupations & -0.0219 & -0.0559 \\
\hline Post-CovidxPercent Management Occupations & -0.0287 & -0.0314 \\
\hline Post-CovidxPercent Building and Grounds Cleaning and Maintenance Occupations & -0.0700 & 0.00725 \\
\hline Post-CovidxPercent Community and Social Service Occupations & -0.214 & -0.199 \\
\hline Post-CovidxPercent Business and Financial Operations Occupations & $-0.324^{* * *}$ & $-0.303^{* *}$ \\
\hline Post-CovidxLog Median Income & $-0.0148^{* *}$ & $-0.0145^{*}$ \\
\hline Post-CovidxPercent age 25-plus with some college degree & $-0.157^{* * *}$ & $-0.136^{* * *}$ \\
\hline
\end{tabular}

Sources: Coefficients from authors' calculations using population data from the ACS and transaction data from Affinity.

\section{B.3 Normalized Coefficients}

Finally, we verify that the ranking of the degree to which occupations are associated with relatively higher consumption spending does not vary substantially once we allow for normalized shares of occupations. Throughout our analysis, we explore how a 1 percentge point (p.p.) additional share of an occupation rather than the omitted occupation affects relative consumption after the onset of the pandemic. An alternative approach that we explore as a robustness check is to normalize the occupation share in a ZCTA. Instead of looking at the shares of occupation in a ZCTA, we 
instead consider the number of standard deviations from the mean of the occupation share across ZCTAs. We replicate regression (1) in Table 5. The results are displayed in Table 11. Regression (1) gives the results for the normalized shares, while regression (2) gives the results for the standard shares. The magnitudes of our coefficients change (by definition), however, the significant variation in spending between occupations remains and the order of occupations (from largest to smallest coefficient) remains largely unchanged. Therefore, normalizing the shares of occupations does not substantially change our results.

We verify that we obtain the same result after normalizing the percentages of each occupation in each ZCTA. To do this, we adhere to the following methodology:

- Calculate $\overline{\text { majorgroup }_{z}}$, that is the average percentage of the labor force in occupation $j$ across all ZCTAs.

- Calculate $s_{j}$, that is the standard deviation of the percentage of the labor force in occupation $j$ across all ZCTAs.

- For a given ZCTA, $z$, calculate normalized $_{z, j}=\left(\right.$ majorgroup $\left._{z, j}-\overline{\text { majorgroup }_{j}}\right) /\left(s_{j}\right)$.

- Then follow the same methodology for regressions explained in Section 3.1 using (postcovid ${ }_{t} \times$ normalized $\left._{z}^{j}\right)$ instead of $\left(\right.$ postcovid $\left._{t} \times \operatorname{majorgroup}_{z}^{j}\right)$.

Table 11. Pre-stimulus Results, Normalized Coefficients

\begin{tabular}{|c|c|c|}
\hline & $\begin{array}{c}\text { Normalized Percents } \\
(1) \\
\text { Log Spend }\end{array}$ & $\begin{array}{c}\text { Regular Percents } \\
(2) \\
\text { Log Spend }\end{array}$ \\
\hline Post-CovidxPercent Office and Administrative Support Occupations & $0.0120^{* * *}$ & $0.329^{* * *}$ \\
\hline Post-CovidxPercent Computer and Mathematical Occupations & $0.00627^{* *}$ & $0.302^{* *}$ \\
\hline Post-CovidxPercent Production Occupations & $0.0106^{* * *}$ & $0.278^{* * *}$ \\
\hline Post-CovidxPercent Installation, Maintenance, and Repair Occupations & $0.0118^{* * *}$ & $0.272^{* * *}$ \\
\hline Post-CovidxPercent Food Preparation and Serving Related Occupations & $0.00776^{* * *}$ & $0.247^{* * *}$ \\
\hline Post-CovidxPercent Transportation and Material Moving Occupations & $0.00946^{* * *}$ & $0.230^{* * *}$ \\
\hline Post-CovidxPercent Architecture and Engineering Occupations & $0.00415^{*}$ & $0.218^{*}$ \\
\hline Post-CovidxPercent Legal Occupations & $0.00566^{*}$ & $0.211^{*}$ \\
\hline Post-CovidxPercent Arts, Design, Entertainment, Sports, and Media Occupations & $0.00635^{* *}$ & $0.190^{* *}$ \\
\hline Post-CovidxPercent Healthcare Practitioners and Technical Occupations & $0.00771^{*}$ & $0.183^{*}$ \\
\hline Post-CovidxPercent Healthcare Support Occupations & $0.00459^{*}$ & $0.171^{*}$ \\
\hline Post-CovidxPercent Farming, Fishing, and Forestry Occupations & $0.00678^{*}$ & $0.150^{*}$ \\
\hline Post-CovidxPercent Life, Physical, and Social Science Occupations & 0.00418 & 0.117 \\
\hline Post-CovidxPercent Construction and Extraction Occupations & 0.00342 & 0.108 \\
\hline Post-CovidxPercent Personal Care and Service Occupations & 0.00407 & 0.0931 \\
\hline Post-CovidxPercent Sales and Related Occupations & 0.00406 & 0.0887 \\
\hline Post-CovidxPercent Educational Instruction and Library Occupations & -0.000434 & -0.0219 \\
\hline Post-CovidxPercent Management Occupations & -0.00153 & -0.0287 \\
\hline Post-CovidxPercent Building and Grounds Cleaning and Maintenance Occupations & -0.00139 & -0.0700 \\
\hline Post-CovidxPercent Community and Social Service Occupations & -0.00267 & -0.214 \\
\hline Post-CovidxPercent Business and Financial Operations Occupations & $-0.00743^{* * *}$ & $-0.324^{* * *}$ \\
\hline Post-CovidxLog Median Income & $-0.0148^{* *}$ & $-0.0148^{* *}$ \\
\hline Post-CovidxPercent age 25-plus with some college degree & $-0.157^{* * *}$ & $-0.157^{* * *}$ \\
\hline
\end{tabular}

ZCTA FE, Time FE, Post-CovidxState interaction terms, and Post-CovidxDemographic interaction terms included in all panels

${ }^{*} p<0.05,{ }^{* *} p<0.01,{ }^{* * *} p<0.001$

Sources: Coefficients from authors' calculations using population data from the ACS and transaction data from Affinity.

\section{Spending Category Definitions}

For each spending category we include the following Merchant Classification Codes: 


\section{Grocery:}

- 5411: Grocery Stores and Supermarkets

\section{Food-Out:}

- 5811: Caterers

- 5812: Eating Places and Restaurants

- 5813: Drinking Places (Alcoholic Beverages) - Bars, Taverns, Nightclubs, Cocktail Lounges, and Discotheques

- 5814: Fast Food Restaurants

\section{Recreation:}

- 4457: Boat Rentals and Leasing

- 4468: Marinas, Marine Service, and Supplies

- 7032: Sporting and Recreational Camps

- 7829: Motion Picture and Video Tape Production and Distribution

- 7832: Motion Picture Theaters

- 7841: DVD/Video Tape Rental Stores

- 7911: Dance Halls, Studios and Schools

- 7922: Ticket Agencies and Theatrical Producers (Except Motion Pictures)

- 7929: Bands, Orchestras, and Miscellaneous Entertainers

- 7932: Billiard and Pool Establishments

- 7941: Commercial Sports, Professional Sports Clubs, Athletic Fields, and Sports Promoters

- 7991: Tourist Attractions and Exhibits

- 7992: Public Golf Courses

- 7993: Video Amusement Game Supplies

- 7994: Video Game Arcades/Establishments

- 7995: Betting, including Lottery Tickets, Casino Gaming Chips, Off-Track Betting, and Wagers at Race Tracks

- 7996 Amusement Parks, Circuses, Carnivals, and Fortune Tellers

- 7997: Membership Clubs (Sports, Recreation, Athletic), Country Clubs, and Private Golf Courses

- 7998: Aquariums, Seaquariums, Dolphinariums, and Zoos

- 7999: Recreation Services (Not Elsewhere Classified)

- 7800: Government-Owned Lotteries (US Region only)

- 7801: Government Licensed On-Line Casinos (On-Line Gambling)

- 7802: Government-Licensed Horse/Dog Racing

\section{Utilities:}

- 4812: Telecommunication Equipment and Telephone Sales 
- 4814: Telecommunication Services, including Local and Long Distance Calls, Credit Card Calls, Calls Through Use of Magnetic Stripe-Reading Telephones, and Fax Services

- 4816: Computer Network/Information Services

- 4821: Telegraph Services

- 4829: Money Transfer

- 4899: Cable, Satellite and Other Pay Television/Radio/Streaming Services

- 4900: Utilities - Electric, Gas, Water, and Sanitary

- 5983: Fuel Dealers - Fuel Oil, Wood, Coal, and Liquefied Petroleum

\section{Derivation of the Occupational Unemployment Risk Score}

To create each of our occupational unemployment risk scores, we utilize the following procedure:

1. For occupational unemployment risk score $R$ (either proximity, work-from-home, or consequence of error) and its corresponding set of $\mathrm{O}^{*} \mathrm{NET}$ questions $Q_{R}$, we find $r_{q, o}$, the average response to question $q \in Q_{R}$ for each O*NET-SOC code $o$.

2. We then find the average response per SOC detailed occupation $r_{q, s}=\frac{\sum_{o \in s} r_{q, o}}{n}$, where $s$ is a given SOC detailed occupation, and $\mathrm{n}$ is the number of $o$, such that $o \in s$.

3. Next we use data from the May 2019 Occupational Employment Statistics (OES) data set to find the employment-weighted average score for each SOC major group: $r_{q, m}=$ $\frac{\sum_{s \in m} r_{q, s} * e_{s}}{e_{T}}$, where $\mathrm{m}$ is a given SOC major group, $e_{s}$ is the employment in SOC detailed occupation $s$, and $e_{T}=\sum_{s \in m} e_{s}$.

4. If there are many $q$ such that $q \in Q_{R}$, we then create a new binary variable, $b_{q, m}$, which equals 1 if $r_{q, m} \geq 3.5$, meaning that the majority of respondents to the O*NET Survey Question gave an answer greater or equal to 4 . We then create variable $p_{Q, m}=\frac{\sum_{q \in Q_{R}} b_{q, m}}{n}$.

5. Finally, we calculate the value of score $R$, for each major occupational group. If there are many $q$ such that $q \in Q_{R}$, then $R$ equals the value of $p_{Q, m}$ normalized between 0 and 1 . Otherwise, $R$ equals the value of $r_{q, m}$ normalized between 0 and 1 . Scores are normalized by subtracting the maximum score across all occupations from a given score, and then dividing by the difference between the maximum and minimum scores across occupations.

For each of the occupational unemployment risk scores, we include the following O*NET Survey Questions ${ }^{17}$ :

\section{Cannot Work from Home Score:}

- WA (4A): How important is inspecting equipment, structures, or materials to the performance of your current job?

\footnotetext{
${ }^{17}$ Work context is abbreviated to WC; work activity is abbreviated to WA
} 
- WA (16A): How important is performing general physical activities to the performance of your current job?

- WA (17A): How important is handling and moving objects to the performance of your current job?

- WA (18A): How important is controlling machines and processes to the performance of your current job?

- WA (20A): How important is operating vehicles, mechanized devices, or equipment to the performance of your current job?

- WA (22A): How important is repairing and maintaining mechanical equipment to the performance of your current job?

- WA (23A): How important is repairing and maintaining electronic equipment to the performance of your current job?

- WA (32A): How important is performing for or working directly with the public to the performance of your current job?

- WC (4): How frequently does your current job require electronic mail?

- WC (17): How often does your current job require you to work outdoors, exposed to all weather conditions?

- WC (18): How often does your current job require you to work outdoors, under cover (like in an open shed)?

- WC (14): How often is dealing with violent or physically aggressive people a part of your current job?

- WC (29): How often does your current job require that you be exposed to diseases or infection?

- WC (33): How often does your current job require that you be exposed to minor burns, cuts, bites, or stings?

- WC (37): How much time in your current job do you spend walking or running?

- WC (43): In your current job, how often do you wear common protective or safety equipment such as safety shoes, glasses, gloves, hearing protection, hard hats, or life jackets?

- WC (44): In your current job, how often do you wear specialized protective or safety equipment, such as breathing apparatus, safety harness, full protection suits, or radiation protection?

\section{Proximity Score:}

- WC (21): How physically close to other people are you when you perform your current job?

\section{Low Consequence of Error Score:}

- WC (45): How serious a mistake can you make on your current job (one you can't easily correct)? 